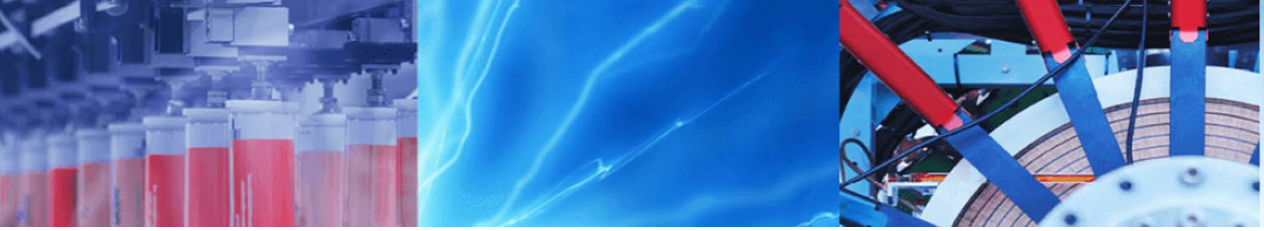

Research Article

\title{
Simulation of pseudo-text synthesis for generating words with long-range dynamic correlations
}

\author{
Hiroshi Ogura $^{1}$ (D) Hiromi Amano ${ }^{1} \cdot$ Masato Kondo $^{1}$
}

Received: 15 October 2019 / Accepted: 1 July 2020 / Published online: 16 July 2020

(c) Springer Nature Switzerland AG 2020

\begin{abstract}
In a previous study, we have regarded real written texts as time series data and have tried to investigate dynamic correlations of word occurrences by utilizing an autocorrelation function (ACF). The results showed that the obtained ACFs can be classified into two groups: One was a group of ACFs showing dynamic correlations and the other was a group of ACFs showing no correlations. A word having the former ACF was called Type-I word, and a word with the latter ACF was called Type-II word. In this study, we investigate the mechanism of generating dynamic correlations of Type-I words by conducting simulations of pseudo-text synthesis. The results of the simulation clarified that inheritance of words between subsequent paragraphs is key to generate dynamic correlations of Type-I words, indicating a important role of long-range memory with duration time from few to several tens of sentences.
\end{abstract}

Keywords Autocorrelation function · Stochastic process · Text synthesis · Simulation · Long-range correlation

\section{Introduction}

During the last decades, it has been clarified that dynamic correlations in written texts are universally observed at any scale of language hierarchy (letters, syllables, words, sentences, etc.) when we regard written texts as time series data ([1-3] and references cited therein). Since our thoughts or ideas are multidimensional complex objects, the reduction in the multidimensional space is inevitable to express the thoughts/ideas as one-dimensional streams of words in written texts. During the reduction process, we use memory to create texts and this is considered to be the origin of observed dynamic correlations [1].

Various efforts to detect and to characterize the dynamic correlations in written texts and also efforts of developing mathematical models of these correlations have been attempted [1, 2, 4-10]. Among these, a recurrence time analysis accompanied with word-level mapping $[8,10]$ is attractive because of the following reasons. The word-level mapping converts given sequence of words into time series data in the way that all the words are enumerated in order of appearance, as $i=1,2, \ldots, N$, where $i$ plays the role of the time along the text having $N$ total number of words. Therefore, without any additional manipulations, the word-level mapping scheme offers time series data of occurrences of a considered word. By using this scheme, Altmann et al. [8] showed that the recurrence time of words (time interval between the occurrences of a considered word) is distributed in accordance with the Weibull distribution in various corpora, and Tanaka-Ishii and Bunde [10] extended Altmann's method to rare words and found that $S_{Q}(r)$, which is the probability of recurrence time of a rare word being larger than $r$, also follows the Weibull distribution. These two studies achieve significant advances in the sense that they provide the mathematical method of quantifying word

Hiroshi Ogura, ogura@cas.showa-u.ac.jp; Hiromi Amano, kayanm@cas.showa-u.ac.jp; Masato Kondo, mkondo@nr.showa-u. ac.jp | ${ }^{1}$ Department of Information Science, Faculty of Arts and Sciences, Showa University, 4562 Kamiyoshida, Fujiyoshida City, Yamanashi 403-0005, Japan. 
occurrences in literary texts. However, when we intend to analyze dynamic correlations of words in time range within several tens of sentences, the methodology suggested in these studies is not applicable because the validity of their method is restricted in analyzing long-range dynamics timescales of which are longer than several tens of sentences or is restricted for analyzing rare words. The reason for the restriction is that a corresponding stochastic process that brings the Weibull distribution of the recurrence time is an inhomogeneous Poisson process [11, 12]. Therefore, the methodology is adequate only when the independence between any two non-overlapping time intervals is assured. This condition is common to all inhomogeneous Poisson processes $[13,14]$ and approximately holds when we consider long-range dynamics in which we can regard a sufficiently long time interval as a unit time. This is the reason why, in Altmann's work (Fig. 1 in [8]), the fitted curves systematically deviate from observed data in the small time region of $t<100$, although the fitting is good in the larger $t$ region up to $t=10^{4}$. If we intend to analyze dynamic correlations of a word which plays a central role in expressing author's idea, it is obvious that dynamic correlations in the time region within several tens of sentences are specifically important because most ideas are described within such length of sentences. In a previous study [15], to investigate dynamic correlations of words observed in that time range, we used a set of serial sentence numbers assigned from the first to the last sentence in a given text as a discretized time. This time unit allows us to define an appropriate autocorrelation function (ACF) for words exhibiting dynamic correlations, i.e., the ACFs thus defined can capture characteristic behaviors of dynamic correlations which gradually decreases as time increases and such behaviors are common in typical relaxation phenomena in complex systems. Identifications of such characteristic behaviors cannot be achieved by the use of the recurrence time analysis accompanied with the word mapping scheme.

We calculated ACFs for frequent words that appear in 12 famous books and found that the frequent words can be classified into two groups called Type-I and Type-II words in terms of their ACFs [15]. Type-I words are the words showing dynamic correlations and their ACFs, which are gradually decreases as mentioned above, are proved to be perfectly described by the use of a modified Kohlrausch-Williams-Watts (KWW) function. Note that although the KWW function is remarkably similar to the expression of the Weibull distribution, it does not describe a probability distribution, but directly describes dynamics of word occurrences. In other words, the KWW function expresses how correlation of word occurrences decays along time. Type-Il words are the words showing no dynamic correlations and their ACFs are modeled by a simple step-down function. As intuitively expected, it has been shown that the functional form of ACFs of Type-Il words is theoretically derived under the assumption that the stochastic processes governing occurrences of Type-II words are the Poisson point processes [15].

We also found that occurrence patterns of TypeI words are completely different from those of Type-II words. Type-I words occur multiple times in a text in a bursty and context-specific manner, and therefore, they are considered to play important roles for describing an idea or topic. Thus, they are expected to be highly correlated with a duration of, typically, several tens of sentences in which the idea or topic is described. In contrast, Type-II words are not context-specific and their appearance is governed by chance.

At this stage, despite the importance of Type-I words, the stochastic process yielding them has not been clarified except a formal modeling by the use of an additive binary Markov chain [16]. This is mainly because known models to yield ACFs of KWW type are mostly developed in the field of condensed matter physics [17-19], and therefore, they are based on different situations from ours. In this study, we present simulation algorithms which are intended to simulate conversion process of author's thoughts/ideas into a linear sequence of words. The suggested algorithms do not have direct links to the stochastic process governing the generations of Type-I words. However, the algorithms are valuable because they have ability to generate texts containing Type-I words, and thus, they offer the fundamental information of how memory works at generating Type-I words. The information clarified in this study will be served when we construct a more realistic stochastic model for Type-I word generation.

The remainder of this paper is organized as follows. In the next section, we summarize the results of our previous study for later reference. In Sect. 3, we describe our simulation algorithm of pseudo-text synthesis through which we attempt to generate words exhibiting dynamic correlations. Results of our simulation are shown in Sect. 4 where we confirm that characteristic behaviors of ACFs of actual Type-I words are successfully reproduced by our simulation. In the final section, we give our conclusions.

\section{Characteristics of ACFs for Type-I and Type-II words}

\subsection{Definition of ACF}

As mentioned before, we use the set of serial sentence numbers as discretized time. The signal representing a word occurrence/non-occurrence is then defined as 

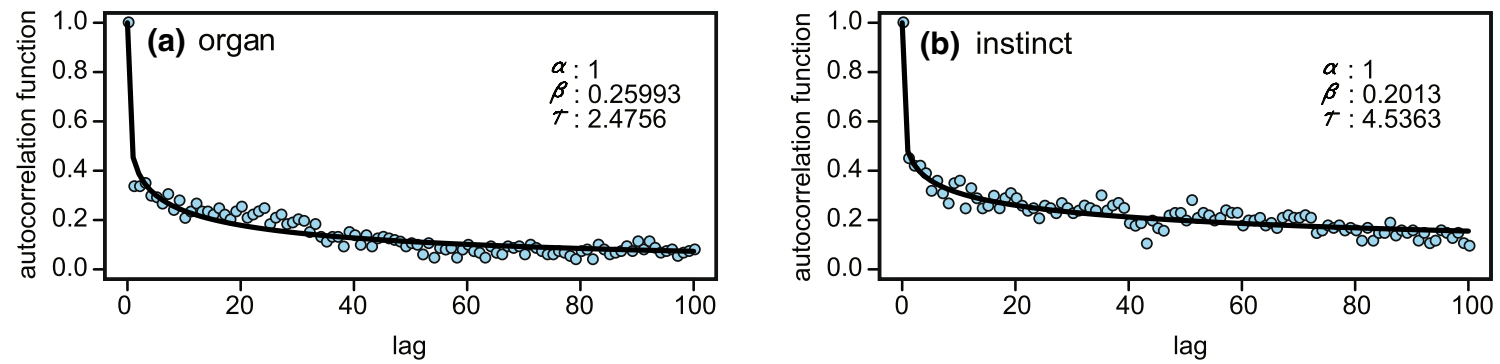

Fig. 1 Examples of ACFs of Type-I words exhibiting strong dynamic correlations. They are ACFs of the words a "organ" and $\mathbf{b}$ "instinct," which are picked from the set of frequent words of Darwin text
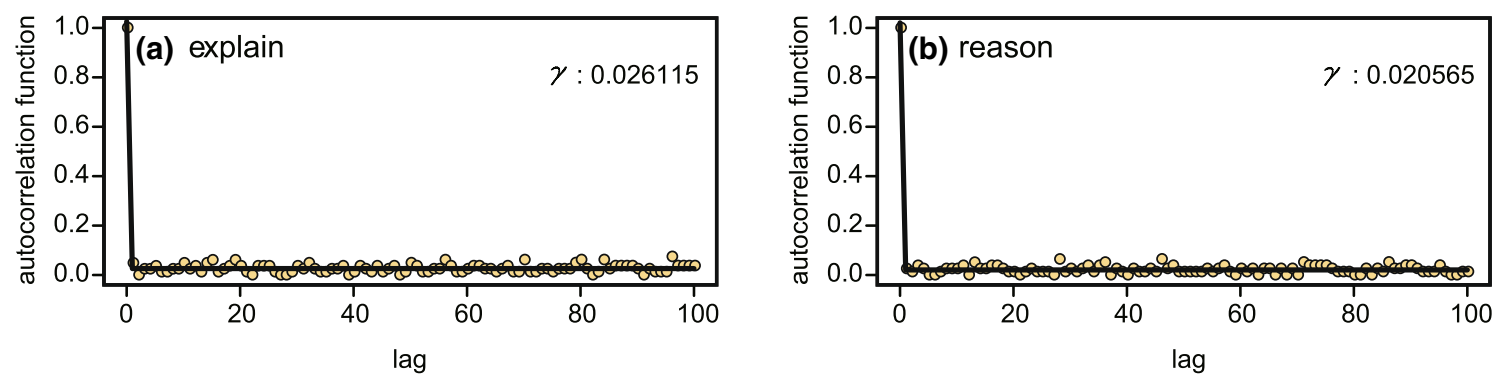

Fig. 2 Examples of ACFs of Type-II words exhibiting no type of correlations. They are ACFs of the words a "explain" and $\mathbf{b}$ "reason," which are picked from the set of frequent words of Darwin text

$A(t)=\left\{\begin{array}{l}1 \text { (if a given word occurs in the th sentence) } \\ 0 \text { (if " does not occur in the } t \text { th sentence) }\end{array}\right.$

From this signal, we calculate the ACF of word occurrences as follows. The starting point is the definition of ACF and their normalized expression widely used in the signal processing area [20, 21]:

$C(t)=\lim _{T \rightarrow \infty} \frac{1}{T} \int_{0}^{T} A(\tau) A(\tau+t) d \tau$,

$\Phi(t)=\frac{C(t)}{C(0)}=\frac{\lim _{T \rightarrow \infty} \frac{1}{T} \int_{0}^{T} A(\tau) A(\tau+t) d \tau}{\lim _{T \rightarrow \infty} \frac{1}{T} \int_{0}^{T} A(\tau) A(\tau) d \tau}$

Intuitively, $C(t)$ and $\Phi(t)$ measure what extent the time varying signal $A(\tau)$ resembles itself at two different time points separated by time $t$. Since our signal is restricted to take only two values 0 and 1 as defined by Eq. (1) and the time is discretized, Eqs. (2) and (3) are proved to be modified as [15]:

$C(t)=\frac{1}{N-t} \sum_{j=1}^{m} A\left(p_{j}+t\right)$,
$\Phi(t)=\frac{N}{m(N-t)} \sum_{j=1}^{m} A\left(p_{j}+t\right)$,

where $N$ is the number of sentences of a considered text, $p_{j}$ the ordinal sentence number at which a considered word occurs and $m$ the number of total occurrences of a considered word in a text. Hereafter, we use Eq. (5) for calculating normalized ACFs.

\subsection{Examples of ACFs for Type-I and Type-II words}

Figures 1 and 2 show typical ACFs of Type-I and Type-II words, which are taken from Charles Darwin's famous work, On the Origin of Species. As we can see in Fig. 1, ACFs of Type-I words exhibit dynamic correlations which persist during several tens of sentences where they gradually decrease. Contrary to this, ACFs of Type-II words suddenly drop at $t=1$ as shown in Fig. 2, indicating that stochastic processes that generate Type-II words are memoryless Poisson point processes.

\subsection{Curve fitting using model functions}

We have attempted to analyze ACFs of Type-I and Type-II words through curve fitting procedure by the use of model 
functions. The model function used for Type-I word ACFs is a modified Kohlrausch-Williams-Watts (KWW) function,

$\Phi_{\mathrm{kwW}}(t)=\alpha \exp \left\{-\left(\frac{t}{\tau}\right)^{\beta}\right\}+(1-\alpha)$,

where $\alpha, \beta$ and $\tau$ are fitting parameters which satisfy inequality conditions; $0<\alpha \leq 1,0<\beta \leq 1$ and $0<\tau$. The original KWW function has been widely used to describe relaxation phenomena in complex systems [17-19, 22], and the modified version, Eq. (6), can also successfully describe decays of dynamic correlations of Type-I words as seen in the solid lines of Fig. 1. The model function used for describing Type-II word ACFs is a simple stepdown function;

$\Phi_{\text {Poisson }}(t)= \begin{cases}1 & (t=0) \\ \gamma & (t \geq 1),\end{cases}$

where $\gamma$ is a fitting parameter satisfying $0<\gamma<1$. As indicated by solid lines in Fig. 2, Eq. (7) is sufficient to express ACFs of Type-II words which do not have any kind of dynamic correlation. The most significant result clarified in our previous study [15] is that all the frequent words in 12 famous books are well classified as Type-I or Type-II without any exceptions. Here, the frequent words mean the words that appear at least 50 sentences over a considered text.

\subsection{Comparison of Type-I and Type-II words in word occurrence signal}

To obtain intuitive understanding of difference between Type-I and Type-II words, we compare their characteristic behaviors in occurrence signals. Figure $3 a$ and $d$ shows signals of word occurrences, $A(t)$ defined by Eq. (1), for typical Type-I and Type-II words, respectively. Figure $3 \mathrm{~b}$ and e indicates cumulative count of word occurrences through text for corresponding words. As clearly shown in Fig. 3a and b, the rate of word occurrences frequently changes with various burstiness level for the Type-I words. On the other hand, it does not change for the Type-II words, as shown in Fig. $3 \mathrm{~d}$ and e. This means that the Type-I word occurs multiple times in a text in a bursty and context-specific manner, and is therefore expected to be highly correlated with durations, typically several tens of sentences in which the idea or topic relevant to the word is described. From Fig. 3b, we notice that the duration time of burst state also takes various lengths depending on their burstiness levels. Such a complex dynamic correlation results in the ACF shown in Fig. 3c. Contrast to this, the occurrence rate of the Type-II word is almost constant throughout text, and thus, the word emission is considered to be governed by a homogeneous Poisson point process with a single rate parameter, which brings the ACF shown in Fig. $3 f$.

\subsection{Modeling of waiting time distribution}

As described above, the occurrence patterns of Type-I words are very different from those of Type-II words, and therefore, it is obvious that the stochastic process which governs occurrences of Type-I words is not the homogeneous Poisson point process which is characterized by a single rate constant. In this subsection, we try to check whether or not the stochastic process governing generations of Type-I words can be described by a superposition of homogeneous Poisson point processes. We use the superstatistical approach for this purpose.

One major trend for describing complex systems is to adopt the superstatistical approach, which recently becomes a perspective tool in physics, engineering, biology and other fields [23-25]. The basic idea of the superstatistical approach is to describe a considered complex system as a superposition of distributed statistics each of which represents a locally equilibrated subsystem. For analyzing counting processes as in the case of word occurrences along a text, the approach tells us that the process can be considered as a superposition of various Poisson point processes each of which has its own rate constant. Therefore, the waiting time distribution, i.e., the distribution of inter-occurrence times, is given by a superposition of exponential distribution as in the form [25]

$p\left(t_{w}\right)=\int_{0}^{\infty} p(\beta) \beta \exp \left(-\beta t_{w}\right) d \beta$,

where $\beta$ is a rate parameter which characterizes a corresponding Poisson point process and $p(\beta)$ is a probability density function of the rate parameter. When we assume that $p(\beta)$ is given by gamma or Chi-square distribution, which is a reasonable assumption in a sense that their supports are limited to be positive values, then the waiting time distribution is given by the so-called q-exponential distribution [23-25]:

$p\left(t_{w}\right)=(2-q) \lambda\left\{1+\lambda(q-1) t_{w}\right\}^{1 /(1-q)}$.

This distribution is a generalization of the exponential distribution because when we set $q=0$, then the above expression turns out to be

$p\left(t_{w}\right)=\lambda \exp \left(-\lambda t_{w}\right)$,

which is just the exponential distribution and is known to give the waiting time distribution of homogeneous Poisson point process. 
(a)

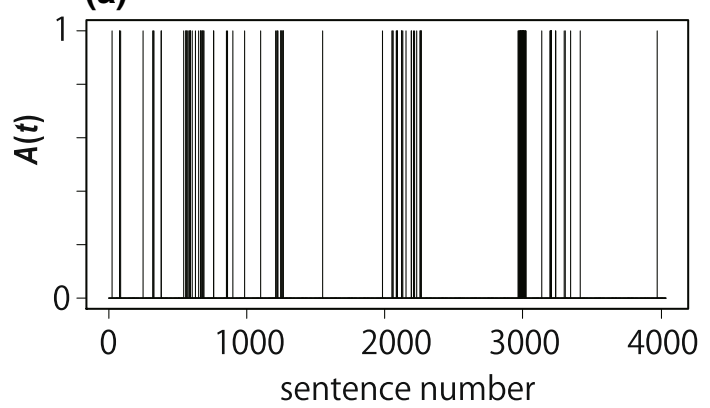

(b)

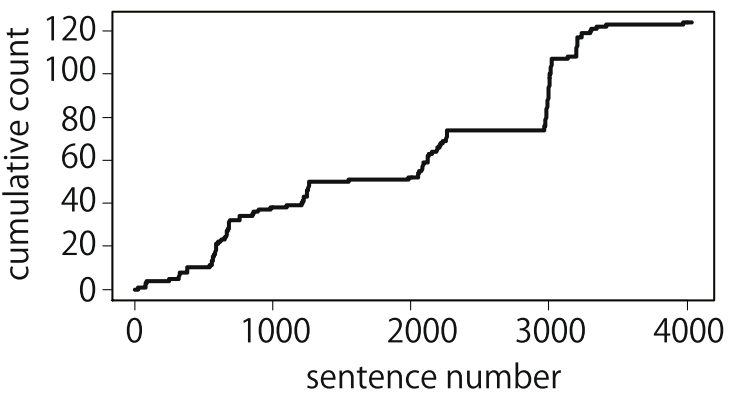

(c)

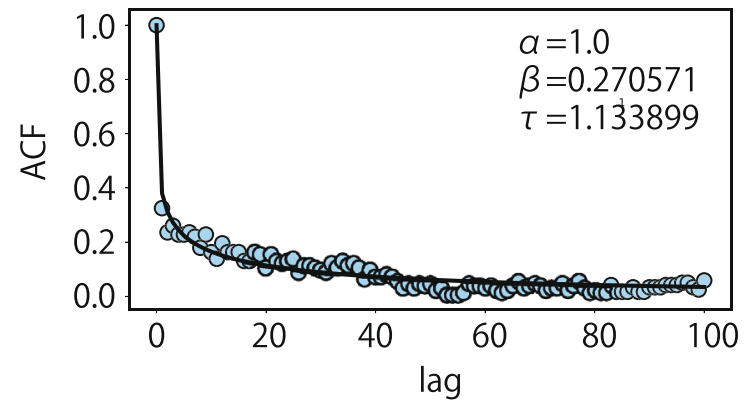

Fig. 3 Comparison between Type-I and Type-II words in word occurrence signal and in cumulative count of word occurrences. The left and right columns show results for the word "seed" and those for the word "subject," respectively, which are taken from

Figure 4 shows waiting time distribution of typical TypeI words picked from several texts (solid circles) and the best fitted q-exponential (red curves) and exponential (blue curves) distributions. We notice in the figure that the fitting of the q-exponential distribution is better than that of the exponential distribution. However, the former is still not adequate especially at regions of waiting times longer than 10 sentences. On the other hand, as indicated by diamonds in the figure, simulated signals of word occurrences by the use of an additive binary Markov chain model [16] completely reproduce characteristics of real waiting time distributions. Note that the memory effect of word occurrences is taken into account in the additive binary Markov chain model through memory functions which persist several tens of sentences. The results shown in the figure (d) subject

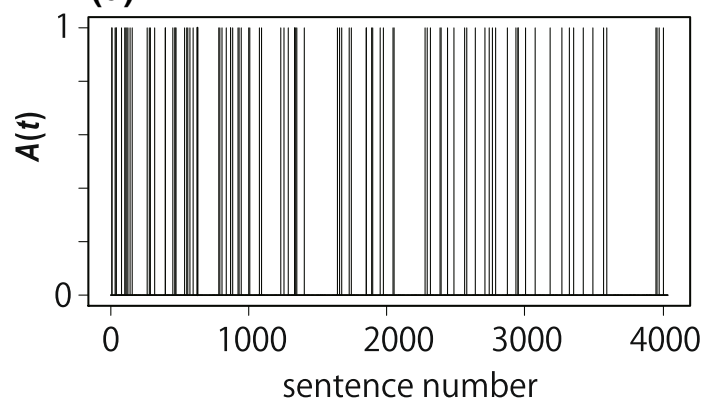

(e)

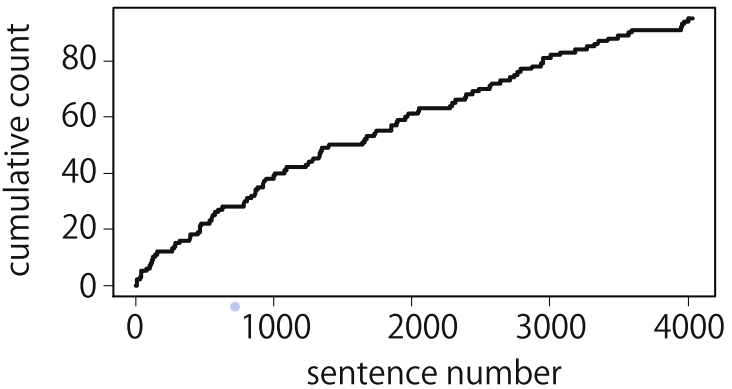

(f)

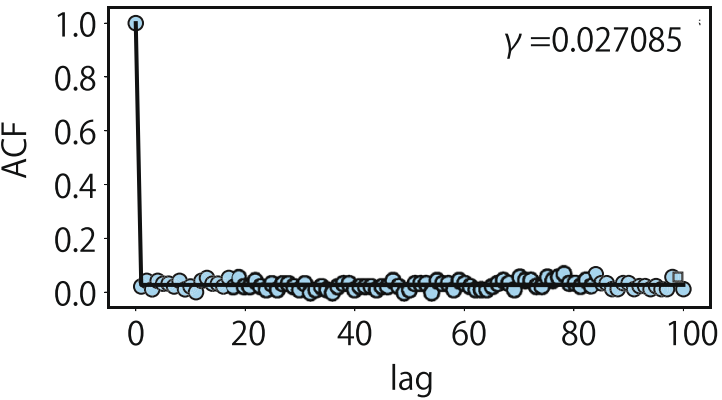

Darwin text. a and d: Word occurrence signal $A(t)$ defined by Eq. (1) through text. $\mathbf{b}$ and e: Cumulative counts of word occurrences through text. c and f: ACFs for "seed" and "subject," making their Type-I and Type-II natures apparent

therefore indicates that observed dynamic correlations of Type-I words cannot be captured by the superposition of Poisson point processes each of which is completely memoryless, but can be captured by introducing memory effects of word occurrences in texts.

\section{$2.6 \beta$ distributions of Type-I words}

Here, we describe how values of the fitting parameter $\beta$ are distributed among ACFs of all Type-I words found in 12 famous books. The parameter $\beta$ is one of the fitting parameters which appear in $\Phi_{\mathrm{kww}}(t)$ (Eq. (6)), and the information of its distribution will be important to discuss the validity of our simulation results in later section. Detailed information of used 12 books is described in "Appendix." 


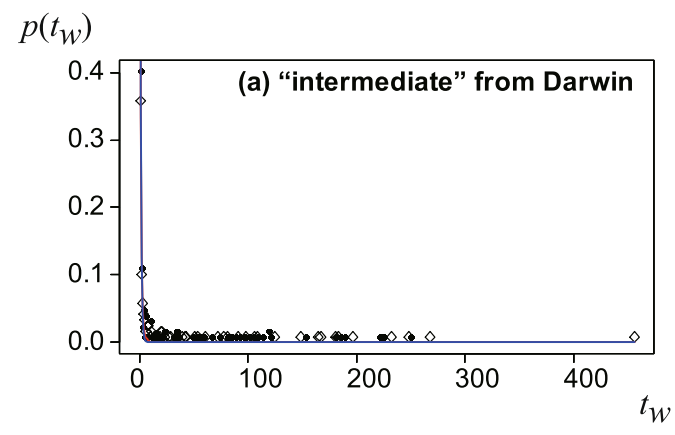

$p\left(t_{w}\right)$
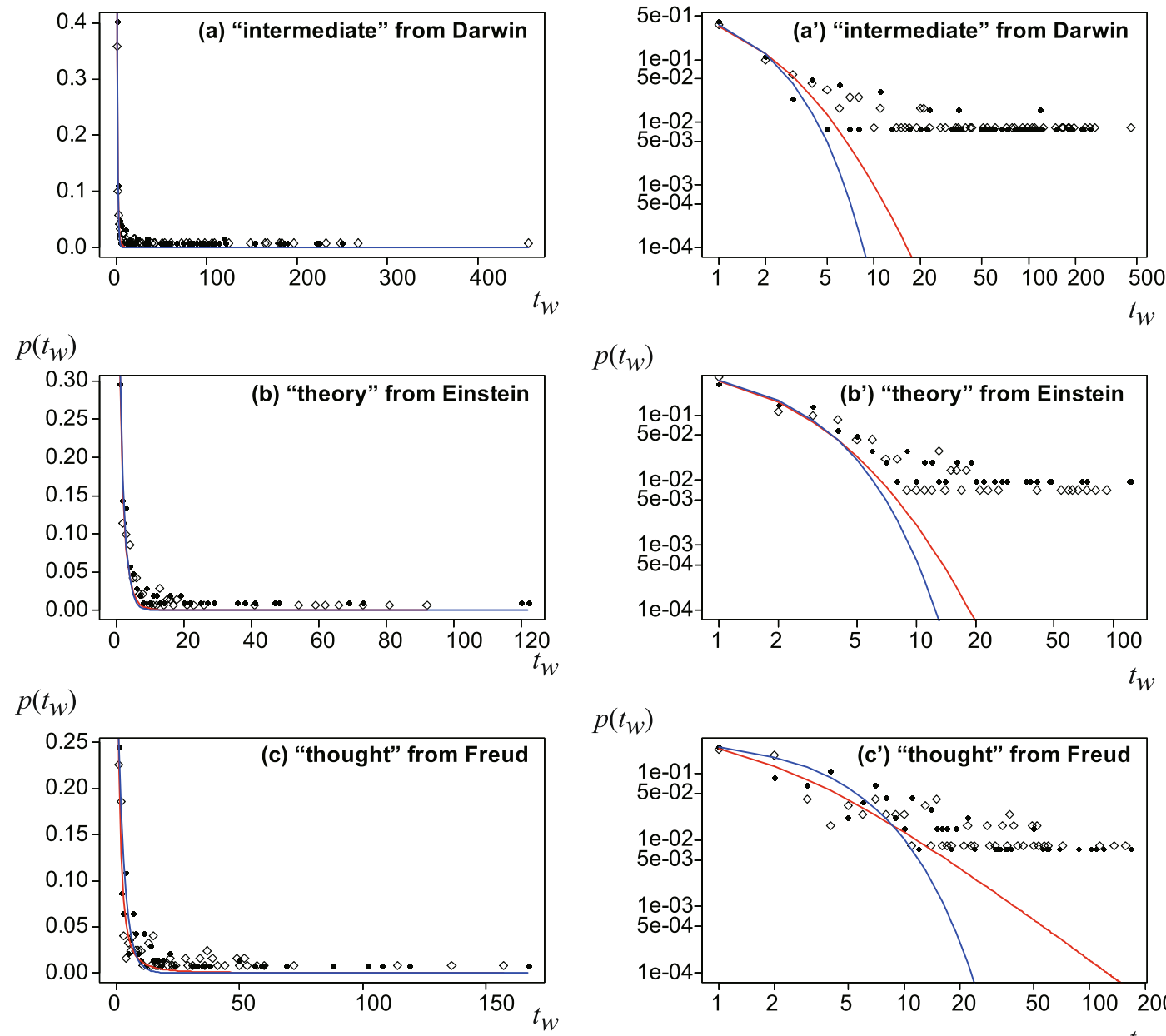

$p\left(t_{w}\right)$
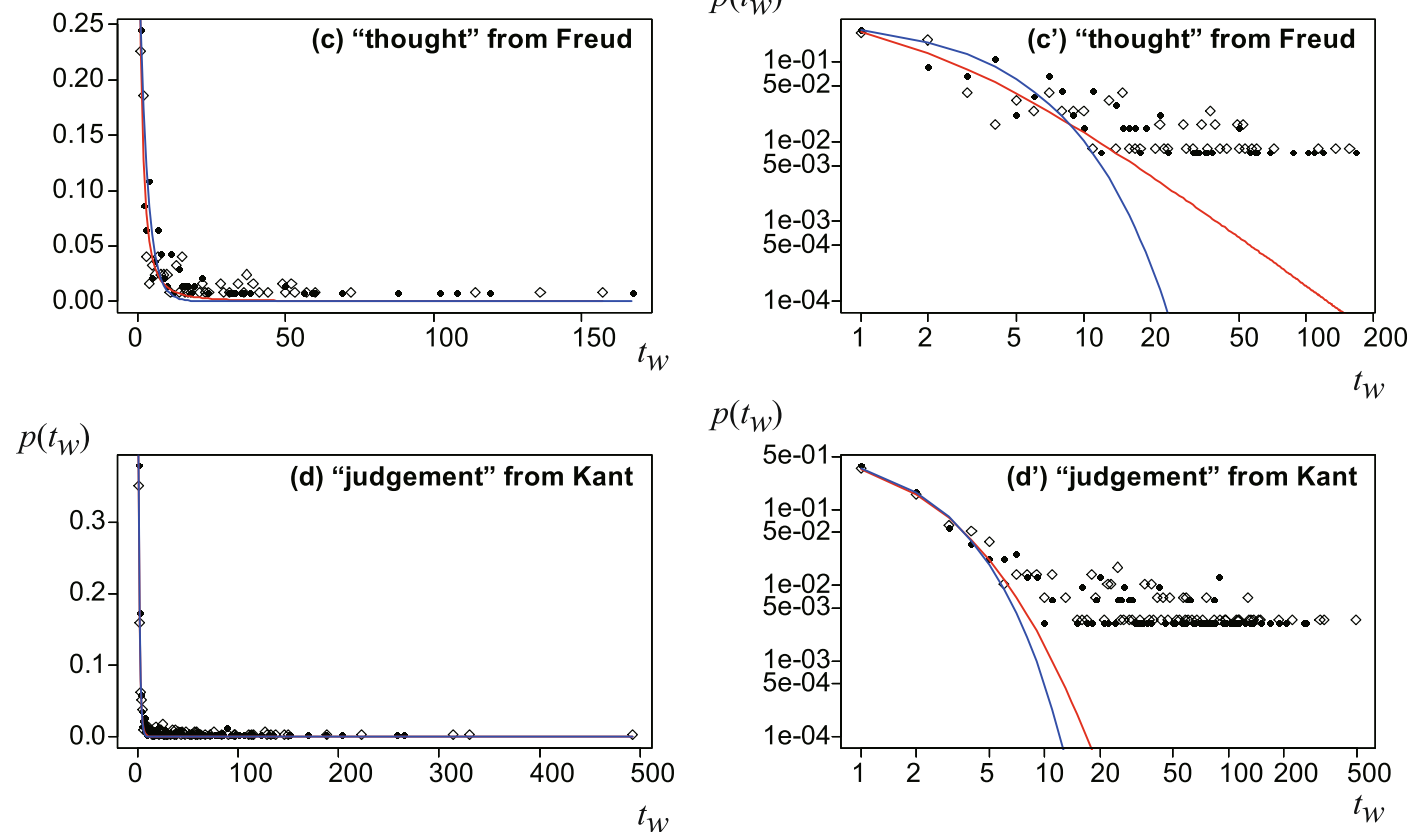

$p\left(t_{w}\right)$

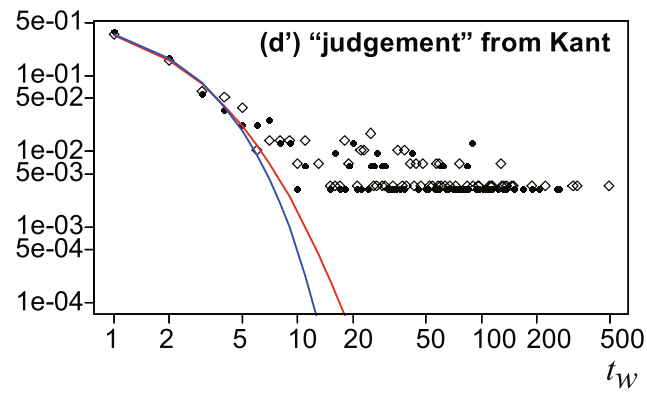

$p\left(t_{w}\right)$

$p\left(t_{w}\right)$
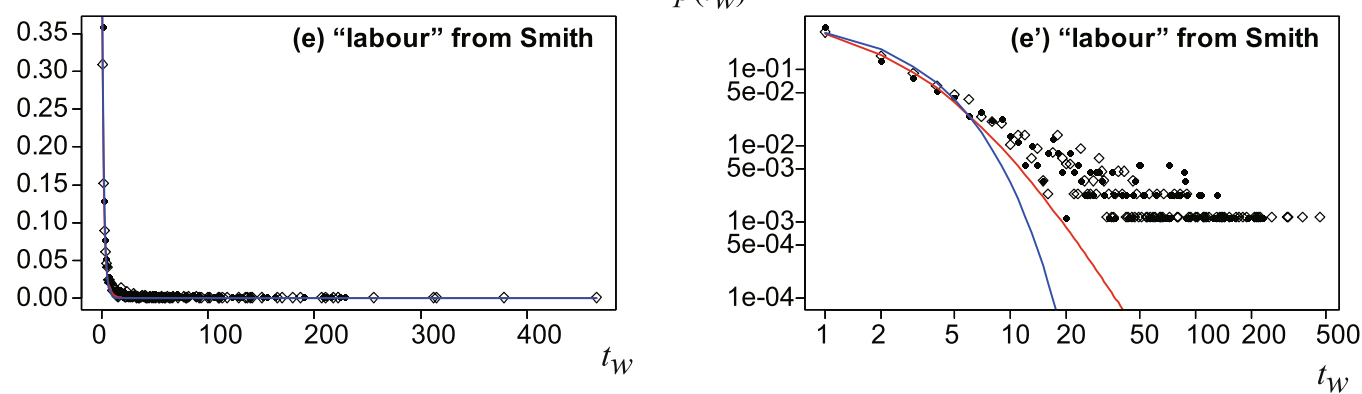
4Fig. 4 Waiting time distributions of typical Type-I words. The left column shows plots on linear scales, while the plots in right column are on logarithmic scales. Solid circles indicate real waiting time distributions observed in written texts, and diamonds indicate simulated distributions by the use of an additive binary Markov chain model [16]. Red lines are the best fitted curves of the q-exponential distributions, and blue lines are those of the exponential distributions. a and a': Results of the word "intermediate" taken from Darwin text, $\mathbf{b}$ and $\mathbf{b}$ ': results of the word "theory" taken from Einstein text, $\mathbf{c}$ and $\mathbf{c}^{\prime}$ : results of the word "thought" taken from Freud text, $\mathbf{d}$ and $\mathbf{d}$ ': results of the word "judgement" taken from Kant text, $\mathbf{e}$ and e': results of the word "labour" taken from Smith text

Figure 5 shows histograms representing distributions of $\beta$ values. In the figure, results of fittings for all of the Type-I words appear in the 12 books are employed. Figure 5 suggests that the typical distribution of $\beta$ is characterized by two components: One is a skewed unimodal distribution peaked around $\beta=0.2$ and ranging from 0.1 to about 0.6. The other is a certain amount of population at $\beta=1.0$. The existence of these two components implies that there are two different mechanisms of generating Type-I words. Actually, the two mechanisms of generating Type-I words will be confirmed later based on simulation results of synthesizing pseudo-texts. In the next section, we will try to construct a simulation model which has an ability to reproduce ACFs with typical values of $\tau$ and $\beta$ observed for real Type-I words.

\section{Simulation algorithms}

In this section, we try to simulate the generation of Type-I words through a pseudo-text synthesis, in which the process of converting our thoughts or ideas into a text consisting of linear sequence of words is taken into account. The conversion model described below is probably the possible simplest one which has a capability of yielding the characteristic behaviors of Type-I ACFs for some of the words appearing in the text. We believe, however, that the origin of the dynamical correlations of Type-I words is captured in our model and the basic mechanism of emerging dynamical correlations involved in the model is common to all real texts.

\subsection{DAG representation and DAG to tree conversion}

The first assumption used in our model is that an idea that an author wants to describe in a text can be schematically expressed by a simple directed acyclic graph (DAG). As depicted in Fig. 6, a DAG is a directed graph with no directed cycles, consisting of nodes and edges, with each edge directed from one node to another. In modeling an idea as a DAG, each node represents one topic, which corresponds to a paragraph or a section or a subsection in the resultant text. We tentatively considered DAGs with 400 nodes to generate text having a realistic number of sentences, with "realistic" indicating comparable to the numbers of sentences in the 12 texts which are listed in "Appendix." Of course, a DAG is too simple for some complex ideas, but at least it is far more complex than the linear one-dimensional stream of topics in written text, and thus is useful to express some ideas. Another important point of our modeling is that DAGs are tractable. Specifically, the notion expressed by a DAG can be transformed into a text in a unique manner, as will be described below.

The author must transform a DAG which represents his notion/idea into a linear one-dimensional sequence of the nodes to determine the order of topics that he wants to describe. Our next assumption is that the transformation can be simulated by the following steps.

1 The DAG is converted to a tree object by the use of a node duplication method [26].

2 The resultant tree obtained at step 1 is converted to a sequence of nodes by the use of the depth-first search algorithm [27]. That is, the order in which the nodes are visited by the algorithm is used as the sequence of nodes which gives the order of topics to be described.

In the following, we describe the meaning and some details of these two steps. The node duplication method used in step 1 is schematically illustrated in Fig. 7. The DAG to tree conversion by the use of the node duplication method is needed to simulate interim progress of our thought process in which we try to simplify hierarchically organized and mutually related topics described by a DAG into an expressible one-dimensional structure. As shown in Fig. $7 a$, if the topic $D$ is derived from a parent topic $B$ and it is also derived from another parent topic $C$, then the simplest way to convert the structure into the tree is to duplicate topic $D$ as shown in Fig. 7b. An intuitive toy example

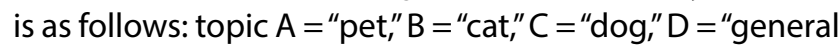
characteristics," $\mathrm{E}=$ "behavior," $\mathrm{F}=$ "food." When we describe the notion "pet" represented by the DAG, then we want to expand the DAG diagram of Fig. 7a to the tree structure, Fig. $7 b$, to consider more detailed content for each topic. The node duplication method depicted in Fig. 7 assures us that DAGs can always be converted into tree structures. The concrete algorithm of the node duplication method used in our simulations will be described in Sect. 3.2.

Another example of the DAG to tree conversion is depicted in Fig. 8, which shows the resultant tree structure converted from an original DAG of Fig. 6 . Note that the tree of Fig. 8 has 11 nodes, while the original DAG of Figs. 6 has 8 nodes, which is the result of the node duplication. Once, the author obtains a tree structure as in Fig. 8, to determine the order of topics in text is straightforward because all the topics to be described are listed in the tree structure as nodes. As described in step 2, 
Fig. 5 Histograms representing distributions of Type-I words
Carroll

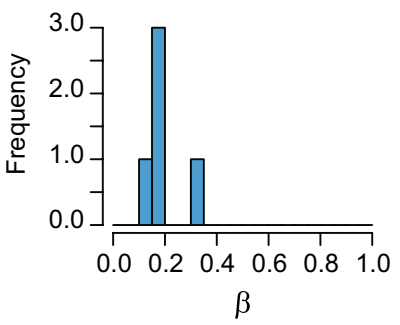

Tolstoy

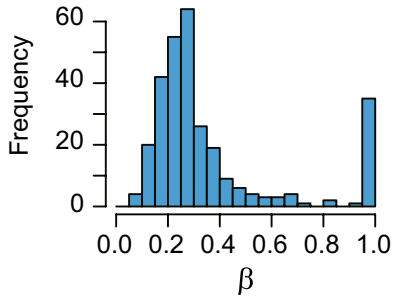

Einstein

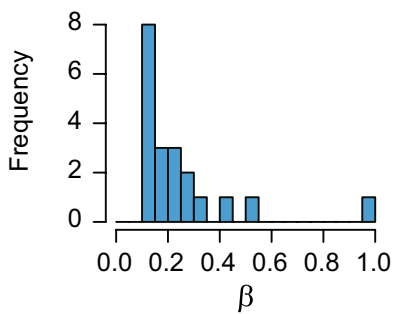

Smith

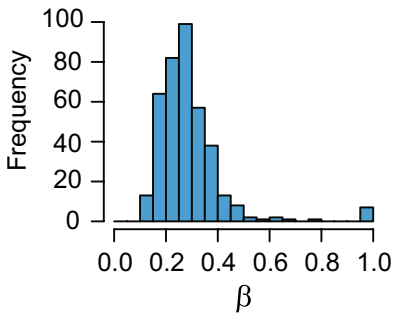

Fig. 6 An example of a DAG with 7 nodes

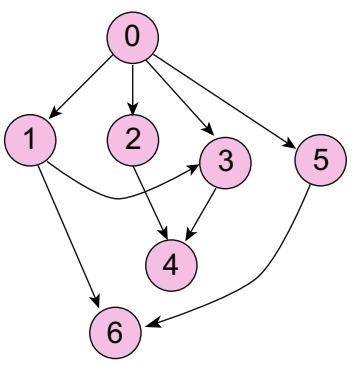

(a)
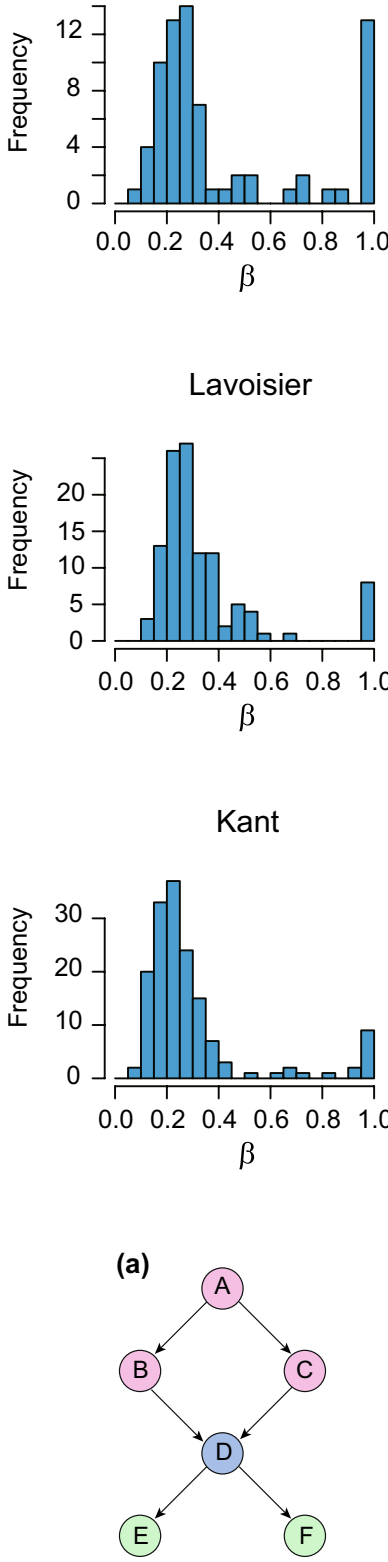

Lavoisier

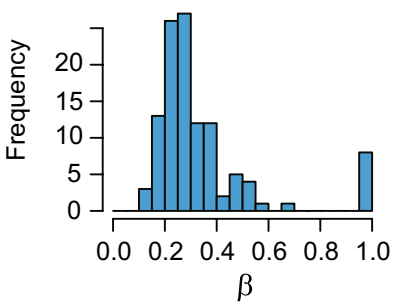

Kant
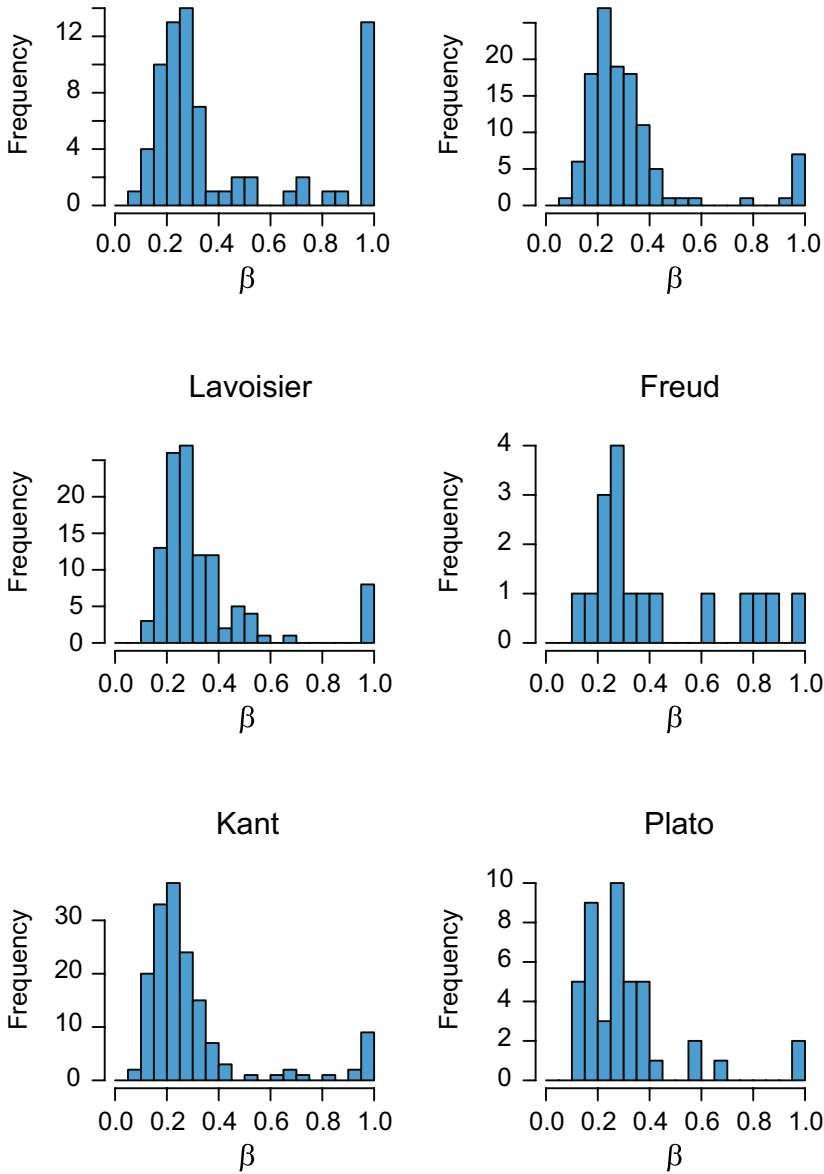

Freud

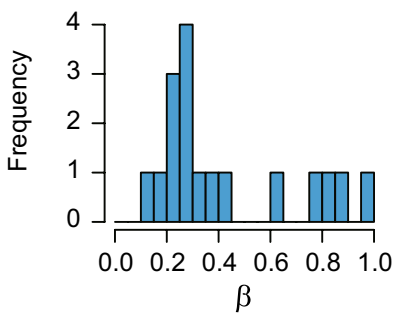

Plato

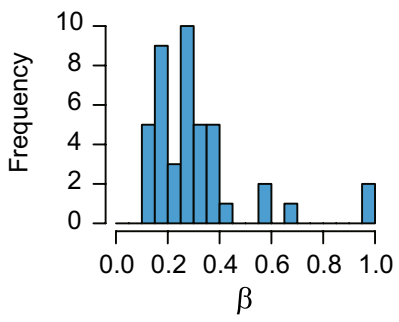

(b)

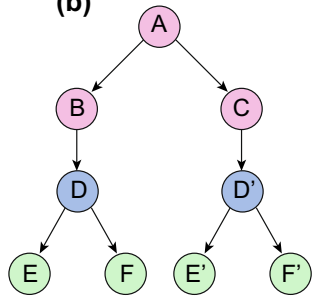

Fig. 7 An example of the DAG to tree conversion using the node duplication method. In a DAG structure (a), node $D$ has two parent nodes $B$ and $C$, and thus, it is duplicated in a tree structure (b) as $D$ '. At the same time, child nodes $E$ and $F$ are also duplicated as $E^{\prime}$ and $F^{\prime}$ in the tree structure (b) 


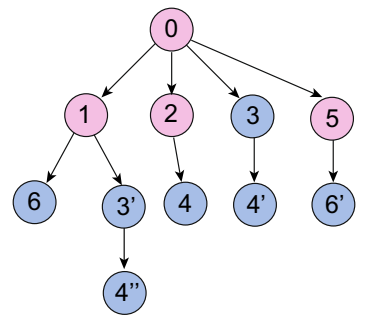

Fig. 8 An example of the tree structure converted from the DAG shown in Fig. 6 by the use of the node duplication method. The corresponding one-dimensional node sequence of this tree given by the depth-first search algorithm is $0 \rightarrow 1 \rightarrow 6 \rightarrow 3^{\prime} \rightarrow 4^{\prime \prime} \rightarrow 2 \rightarrow 4 \rightarrow 3 \rightarrow 4^{\prime} \rightarrow 5 \rightarrow 6^{\prime}$

we assume that the order of nodes, which is equivalent to the order of topics to be described, is given by the depth-first search algorithm [27]. For the tree structure depicted in Fig. 8, this algorithm gives the node sequence as $0 \rightarrow 1 \rightarrow 6 \rightarrow 3^{\prime} \rightarrow 4^{\prime \prime} \rightarrow 2 \rightarrow 4 \rightarrow 3 \rightarrow 4^{\prime} \rightarrow 5 \rightarrow 6^{\prime}$. Although there are several algorithms of tree search each of which enables us to determine a unique sequence of all nodes involved in a tree, the depth-first search gives the most natural order of topics to be described in a text.

By examining the resultant node sequence determined with the depth-first search algorithm, we can recognize one possibility in which the dynamic correlations characterizing Type-I words can be generated. For example, in the node sequence determined from the tree structure of Fig. 8, the node 4 appears three times $\left(4,4^{\prime}\right.$ and $\left.4^{\prime \prime}\right)$ and node 6 two times ( 6 and $\left.6^{\prime}\right)$. These multiple appearances of nodes 4 and 6 are not successive, i.e., they appear again after other nodes appear in the sequence. So, words that are characteristic to the topic of node 4 should be burstly occurred three times and those of node 6 should be burstly occurred twice in a manner that these burst occurrences should be separated with some time interval along the text. Such situations are substantially the same as in the case of Type-I words showing multiple burst occurrences depicted in Fig. $3 a$ and b. Therefore, it is reasonable to expect that our model of text generation yields the Type-I words having dynamic correlations. As will be described later, our model using the DAG to tree conversion indeed shows the ability to yield Type-I words in text, but the obtained Type-I words have the very restricted nature in their ACFs compared with the wide variety of possible ACFs seen in real texts. So, we must add another assumption to our model for generating text in order to extend the ability of yielding various ACFs. It will be turned out later that the needed additional assumption to reproduce various ACFs is an introduction of inheriting words between nodes.

\subsection{Simulation procedures}

Word representation by index To confirm the validity of our conversion model described above, we conducted simulations using the model and have examined whether Type-I words could be involved in generated texts or not. In the simulations, we used a word index instead of using a real word (real text string consists of characters), because we were only interested in the mechanism of generating Type-I words, and hence, it was not necessary to create real written texts for this purpose. We used the word index taking values from 0 to 4999, which means that we fixed the vocabulary size of each text to be 5000 . Conducting the simulations according to the conversion model, we generated pseudo-sentences each of which was a sequence of word indices; this setup was sufficient to calculate ACFs because the value of $A(t)$ defined by Eq. (1) was easily determined whether the considered word index was involved in the th pseudo-sentence or not. ${ }^{1}$

$D A G$ generation In the simulation, we first generated a DAG with 400 nodes. Since the number of nodes were fixed to be 400 , the structure of a DAG was completely determined by identifying all the edges in the DAG. We used the edge matrix representation, em[i][j], for this purpose which has $400 \times 400$ elements $(0 \leq i, j \leq 399)$ and expresses the existence/nonexistence of the edge from a parent node $i$ to a child node $j$ by setting $\operatorname{em}[i][j]=1 / 0$. The algorithm of DAG generation, which is equivalent to how to generate edges among 400 nodes, is shown in Algorithm 1 in the form of pseudo-code.

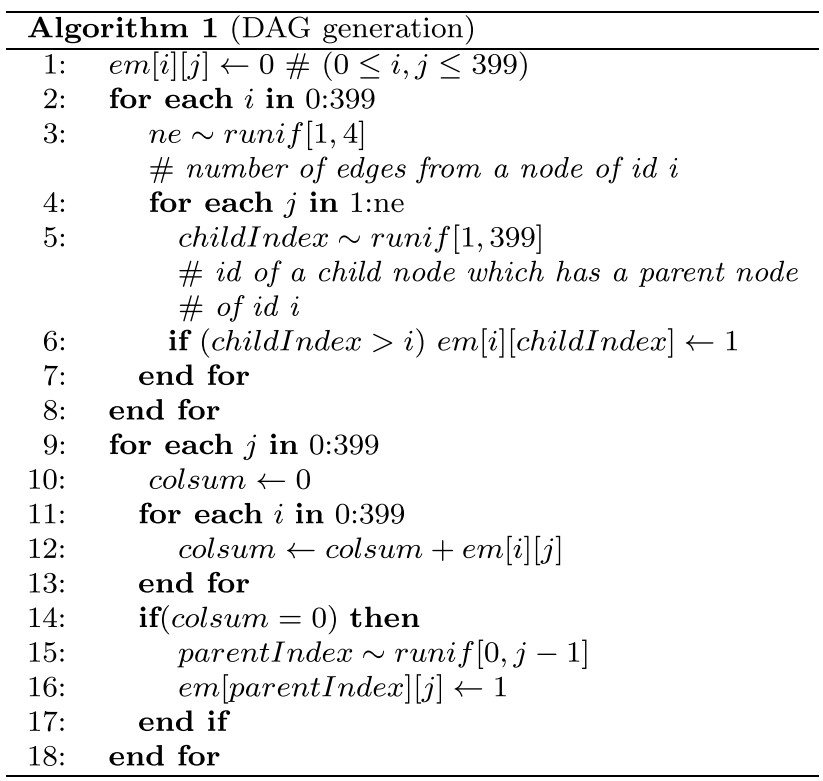

1 Here, we just used the word "pseudo," which means that we use the word indices instead of using real words. In the following, a pseudo-sentence is a sequence of word indices, a pseudo-paragraph is a group of pseudo-sentences, and pseudo-text is a set of pseudo-paragraphs. 
In the algorithm, runif $[m, n]$ means a random number drawn from a discrete uniform distribution ranging from $m$ to $n(m<n)$. Thus, for example, line 3 in Algorithm 1 indicates that the number of possible maximum edges, $n e$, from the parent node $i$ to children is set to be one of the four integers, 1, 2, 3 or 4 . By lines 5 and 6 , the directions of edges were restricted from a node with smaller id to a node of larger id to avoid cyclic connections among nodes. Lines $9 \sim 18$ are needed to assign a parent node to an orphan node which does not have a parent node. Since the direction of edges is restricted from a node with smaller id to a node with larger id as stated above, the root node of any DAGs is always the node with id zero.

Assignment of characteristic words After generating a DAG, we assigned characteristic words to each of the 400 nodes. To do this, we first divided 5000 words into 400 groups each of which had 12.5 words in average, but actually the number of words in each group were randomly determined by the use of a Dirichlet distribution. The algorithm to determine the number of words in each group is shown in Algorithm 2.

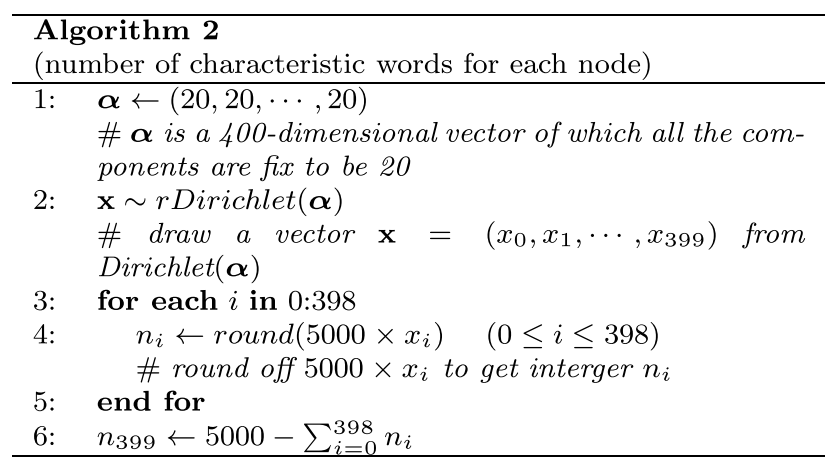

In Algorithm 2, we use the parameters vector $\boldsymbol{\alpha}$ in which all the components are fixed to be 20 so that the Dirichlet distribution with $\boldsymbol{\alpha}$ generates a random vector $\mathbf{x}$ each component of which also takes almost the same value (Chapter 2 of reference [28]). Since the property of the Dirichlet assures that $\sum_{i=0}^{399} x_{i}=1$ (Chapter 2 of reference [28]), the sum of the rounding off of $5000 \times x_{i}$ is approximately 5000 . The adjustment was made at the 400th number, $n_{399}$, so that the sum precisely becomes 5000 .

We then distributed each of the 400 word groups to each of the 400 nodes. This means that each word group is a characteristic word list that characterizes the topic of a corresponding node. The assignment of the characteristic words to a topic/node was made in an ascending manner as follows. For example, if $n_{0}=10$, $n_{1}=12, n_{3}=13, \ldots$ then the characteristic word list (word index list) assigned to the first, second and third nodes is $c w /[0]=(0,1, \ldots, 9), c w /[1]=(10,11, \ldots, 21)$, $c w /[2]=(22,23, \ldots, 34)$, indicating that a node with larger node id has characteristic words of larger word indices. Such assignment is achieved by Algorithm 3 .

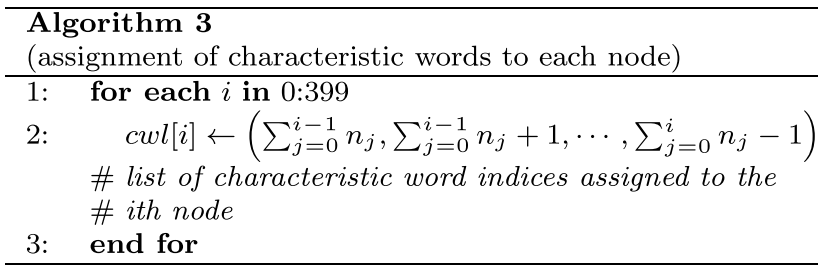

DAG to tree conversion To implement the DAG to tree conversion using the node duplication method, we utilized a table representation which keeps all the node relationships between parents and the corresponding children in a considered DAG or a tree. For clarity and simplicity, we use one example of the conversion to explain the conversion algorithm instead of describing a pseudo-code. The example is shown in Fig. 9 in which the original DAG of Fig. 6 is converted to the final tree structure of Fig. 8 by utilizing the table representation.

Our algorithm first constructs the table representation of a original DAG and then search nodes that have multiple parents in the table. For example, the constructed table and the diagram of step 0 in Fig. 9 represent the same DAG structure, in which there are three nodes, nodes 3, 4 and 6 (A)-I, (B)-I and (C)-I in the table of step 0) that have multiple parents and therefore that are the targets of the node duplication. At step 0 , the first target of the node duplication is node 3 (A)-I) because it has the smallest node id among three target nodes. Thus, the node 3 is duplicated and becomes two nodes, 3 and 3' (A)-II in the table of step 1 ), each of which has one parent node, respectively. Note that the original node 3 in parent node list (B)-I in the table of step 0) must also be replaced with 3 and 3' (B)-II in the table of step 1). In this way, our algorithm duplicates the target node which has multiple parents so that each of duplicated nodes has only one parent, and at the same time, the original target node in the parent list (the second column of the table representation) is also replaced with the duplicated nodes. The second target is node 4 (B)-II) at step 1 which has 3 parent nodes of 2, 3 and 3'. It is thus duplicated as 3 nodes, 4, 4' and 4" (B)-III) at step 2. The third target, node 6 in step 2 (C)-I), is duplicated and becomes 2 nodes (C)-II) at step 3 in the same manner. The algorithm completes the conversion from the original DAG to the tree structure when each of all the nodes in the table has only one parent as step 3 in Fig. 9.

In our simulation of text generation, the number of nodes of the resultant tree should become larger than 400 because of the node duplication. The actual number 
Fig. 9 Algorithm 4 (DAG to tree conversion). Detailed steps of the DAG to tree conversion algorithm using the node duplication method. Step 0 represents the original DAG and its representation in a table form, and step 3 represents the resultant tree and its table representation

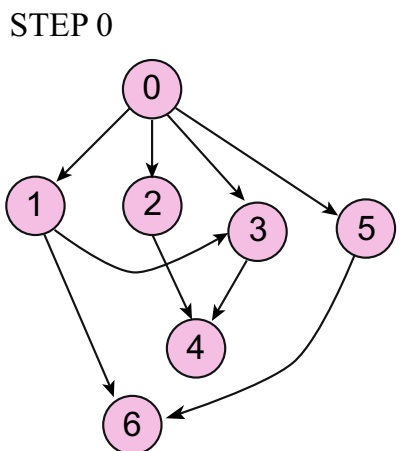

STEP 0

\begin{tabular}{|c|c|}
\hline \begin{tabular}{l|} 
node \\
\end{tabular} & parent node \\
\hline 0 & \\
\hline 1 & 0 \\
\hline 2 & 0 \\
\hline 3 & $\begin{array}{lll}0 & \mathrm{D} & \text { (A)-I }\end{array}$ \\
\hline 4 & 23 (B) $-I$ \\
\hline 5 & 0 \\
\hline 6 & 15 (C) $-I$ \\
\hline
\end{tabular}

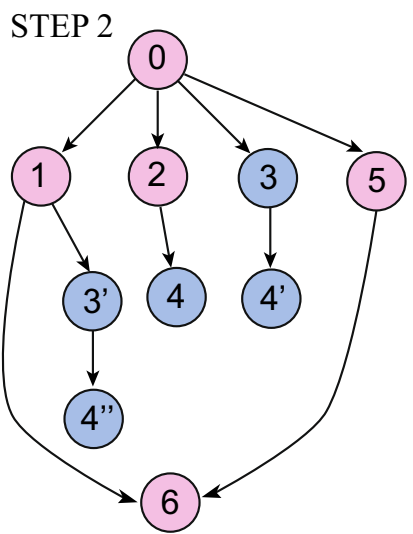

STEP 2
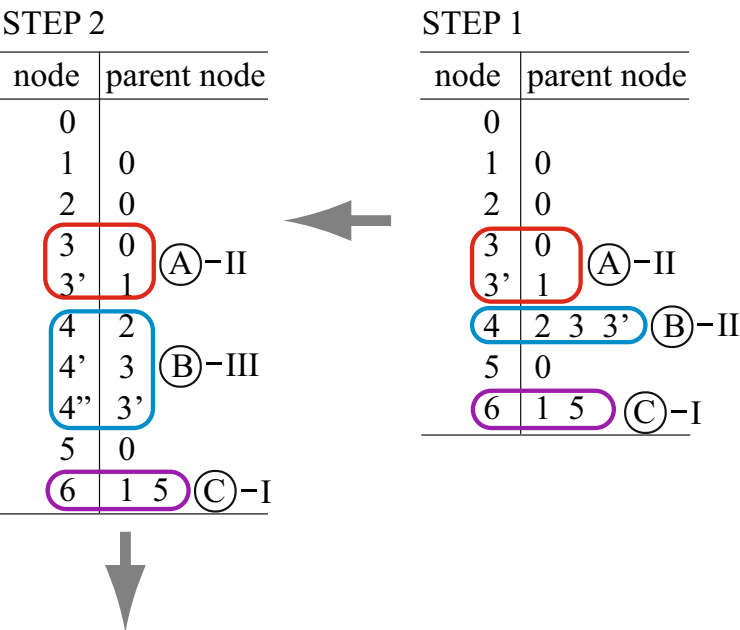

STEP 3

STEP 3

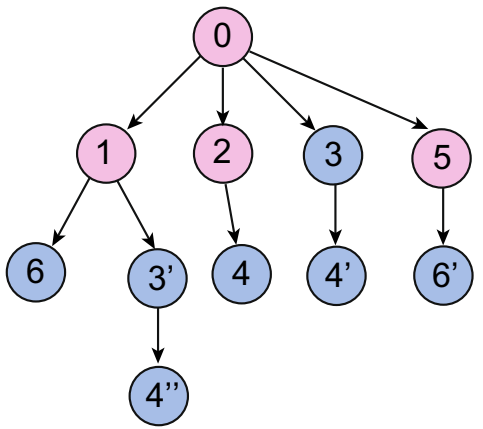

of nodes in the tree was turned out to be ranging from about 800 to about 1400 as shown in Fig. 12, depending on the original DAG structure. At the node duplication, we assumed that the duplicated nodes have the same characteristic word groups with those of original nodes; that is, for the case of step 3 in Fig. 9, the nodes 3 and 3 ' have common characteristic words and this is also holds for the nodes 4, 4' and 4" and for the nodes 6 and 6 '.

Once the tree structure is obtained by the conversion algorithm, one-dimensional node sequence is derived by the use of the depth-first search algorithm as mentioned before.

Synthesis of pseudo-text from node sequence The final procedure of the simulation is to synthesize a pseudotext from the node sequence which provides the order of topics to be described. For simplicity, we always synthesized one pseudo-paragraph for each node/topic. The synthesis was performed along the node sequence; that is, we first generated a pseudo-paragraph corresponding to the first node, which was the root node of id 0 , using the characteristic words assigned to that node, and then, we 
synthesized the second pseudo-paragraph corresponding to the second node in the sequence and so on.

Each pseudo-paragraph corresponding to each node or topic consisted of about 5 pseudo-sentences in average. The number of pseudo-sentences for ith node in the sequence was assigned to a symbol $n p s[i]$ and was randomly drawn from the Poisson distribution with a mean value of 5 according to the following algorithm.

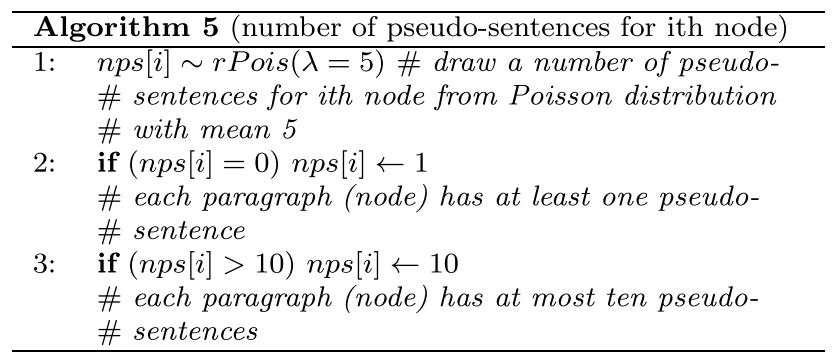

After determining the number of pseudo-sentences in a pseudo-paragraph, we then determined the word length of each pseudo-sentence in the pseudo-paragraph by Algorithm 6 shown below which is similar to Algorithm 5 . In the algorithm, wl[j] means the word length of $j$ th pseudo-sentence in the considered ith pseudo-paragraph.

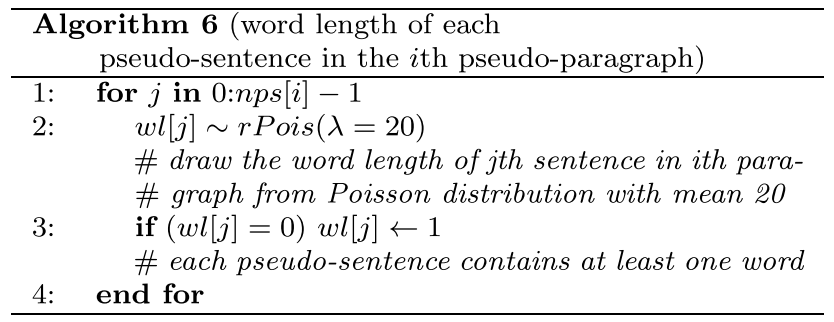

The final part of our simulation of pseudo-text generation is procedures of synthesizing a pseudoparagraph. Consider the ith node in the node sequence. We have assigned $n_{i}$ characteristic words as $c w l[i]=\left(w_{i 0}, w_{i 1}, \ldots, w_{i n_{i}-1}\right)$ to that node by Algorithm 3, have determined the number of pseudo-sentences corresponding to this node/pseudo-paragraph as nps[i] by Algorithm 5 and have determined the word length of each pseudo-sentence as $w /[j](0 \leq j<n p s[i])$ by Algorithm 6 . Given these conditions, we synthesized pseudo-sentences which formed pseudo-paragraph of the ith node by the use of Algorithm 7 which is illustrated in Fig. 10. In the algorithm, we first divided the characteristic words assigned to the ith node into $n p s[i]$ groups. The example shown in the figure is the case where 14 characteristic words $\left(n_{i}=14\right)$ are divided into 5 groups (nps $\left.[i]=5\right)$ that has 2, 2, 3, 4 and 3 words, respectively. The determination of the number of words in each group was made in the same manner as in Algorithm 2 using the Dirichlet distribution. In this case, the parameters vector $\boldsymbol{\alpha}$ is the fivedimensional vector in which all the components are fixed to be 20. The sum of each component in a five-dimensional random vector $\mathbf{x}$ drawn from $\operatorname{Dirichret}(\boldsymbol{\alpha})$ is equal to one, and thus, the round-off of each component of $14 \times \mathbf{x}$ can be used for the number of words in each group. The adjustment was made at the last component of the vector $14 \times \mathbf{x}$ so that the sum of the number of words in each group became 14 .

We next assigned each word group to the each of the 5 pseudo-sentences, $p s[j](0 \leq j<n p s[i]=5)$, as shown in step 1 of Fig. 10. Step 1 is needed to assure that each of the characteristic words assigned to the ith node appears at least one time in the corresponding pseudoparagraph. To complete the pseudo-sentence generation, we then assigned randomly selected words from $c w /[i]$ to each of the pseudo-sentences so that the word length of each pseudo-sentence became predefined number, $w /[j](0 \leq j<n p s[i])$, which has been determined by Algorithm 6 . The selection of each word to be added to a pseudo-sentence at step 2 was made by the use of the discrete uniform distribution so that the probability of selecting each word in $c w /[i]$ was equally likely. When all the $p s[j]$ were generated, they formed the pseudo-paragraph corresponding to the $i$ th node.

An attention must be paid to the point that we did not intend to generate Type-II words through the simulations because the origin of Type-II words has already been clarified in our previous study [15]. If we intend to generate Type-II words through simulations, we must select some amount of words in the whole vocabulary as candidates of Type-II words, and then, we must distribute the selected words into the original 400 nodes in a DAG according to a discrete uniform distribution so that the occurrence of the selected word in each sentence becomes equally likely. However, we distributed each word in the vocabulary as a characteristic word for one of the original 400 nodes as described above, and thus, each word was not common to any two of the original 400 nodes. This distribution policy was chosen because we were not interested in generating Type-II words and the policy helped us to keep the simulation algorithm simple.

\subsection{Non-inheriting and inheriting schemes at generating pseudo-paragraph}

As mentioned earlier, we have used two different algorithms to generate a pseudo-paragraph; one is algorithm 7 shown in Fig. 10 which has already been explained. Although algorithm 7 can generate Type-I words as will be soon shown, the nature of obtained Type-I words was very restricted and failed to reproduce the wide range of 

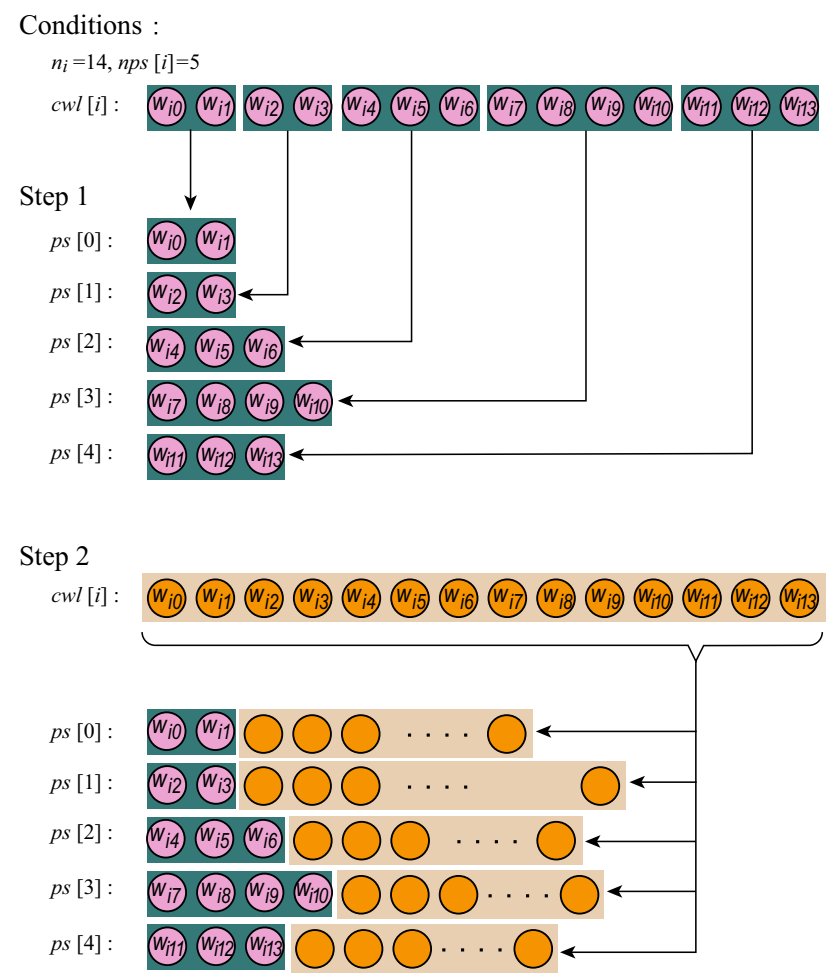

Fig. 10 Algorithm 7 (generation of pseudo-sentences with noninheriting scheme). In this example, the number of characteristic words assigned to the considered ith node is $n_{i}=14$. These characteristic words, $w_{i 0}, w_{i 1}, \ldots, w_{i 13}$ (a set of word indices ranging from $w_{i 0}$ to $w_{i 13}$ ), have already set in the characteristic word list, $c w /[i]$. Five pseudo-sentences, $p s[0], p s[1], \ldots, p s[4]$, are generated through steps 1 and 2 . Each $p s[j](0 \leq j<5)$ is expressed as a list of word indices involved in the sentence. Addition of words to $p s[j](0 \leq j<5)$ at step 2 is made so that the resultant word length of each pseudo-sentence becomes $w /[j]$, which was given by Algorithm 6

fitting parameter $\beta$ which takes values from about 0.1 to 1.0 for Type-I words in real written texts. We thus developed another algorithm through which the characteristic behaviors of Type-I words in real written texts are completely reproduced. This another algorithm is Algorithm 8 illustrated in Fig. 11.

The difference between Algorithms 7 and 8 is as follows. In Algorithm 7, each pseudo-paragraph purely consists of the characteristic words assigned to the corresponding node. On the other hand, in Algorithm 8, each pseudoparagraph consists of not only the characteristic words assigned to the corresponding node, but inherited words from the parent node.

In Algorithm 8, we consider two characteristic word groups for each node, i.e., the inherited words and the characteristic words as shown in the upper part of Fig. 11. The characteristic words are common to those in Algorithm 7 (cw/[i] in Fig. 10 determined by Algorithm 3). They are therefore the words assigned to the considered node

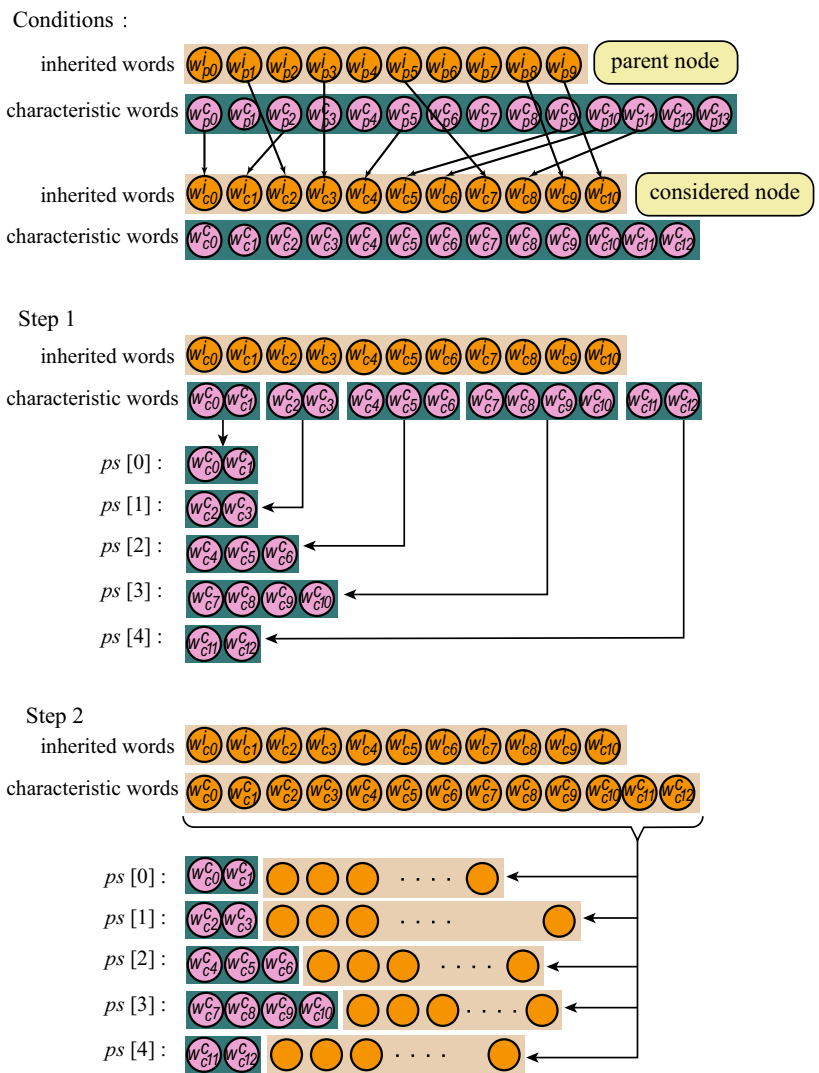

Fig. 11 Algorithm 8 (generation of pseudo-sentences with inheriting scheme). Detailed steps of the pseudo-sentence generation using the inheriting scheme

and sharing with other nodes is prohibited among original 400 nodes in the DAG. ${ }^{2}$ In contrast to this, the inherited words are randomly drawn from the inherited and characteristic word groups of the parent node as shown at the conditions in Fig. 11; consequently, they are shared between the considered and the parent nodes. The number of inherited words was randomly drawn from the Poisson distribution with mean 10 and it was corrected to be one if the drawn number was zero. The selection of words to be inherited from the parent node was set to be equally likely over all inherited and characteristic words of the parent node by the use of the discrete uniform distribution. Here, the parent node does not mean that in the original DAG, but means that in the converted tree structure. Since the parent node of a considered node can be uniquely determined in the tree structure, there is no ambiguity in the procedures of word inheritance described here.

\footnotetext{
2 Note that, however, if the node duplication of a considered node occurs at the DAG to tree conversion, then the characteristic words are also duplicated and the original and duplicated nodes have the same characteristic words.
} 
After setting up the inherited and characteristic words of the considered node, we proceed to step 1 in Fig. 11 which is substantially the same as step 1 of Algorithm 7; in step 1, we distribute all the characteristic words assigned to the considered node to the pseudo-sentences without any overlapping of the words between pseudo-sentences. In step 2, selected words from inherited and characteristic word groups are added to each pseudo-sentence so that the word length of the each pseudo-sentence becomes predefined size. Note that the selection is made from not only the characteristic words, but from the inherited words, which gives rise to the inheritance of words from the parent to children. We used the discrete uniform distribution for selecting word to be added to assure the equal probability of selection over each word in the inherited and characteristic word groups.

As explained above, words can be inherited from parents to children and from children to grandchildren through Algorithm 8. Since we express a topic into text in a manner that the past history of topic descriptions affects contents of the current topic, Algorithm 8 is more natural than the non-inheriting algorithm given by Algorithm 7 . In Algorithm 7, each pseudo-sentence was synthesized from words that were drawn only from the characteristic word group of a considered node. This means that multiple occurrences of a word over different paragraphs/nodes can take place only through the node duplication. On the other hand, in the inheriting scheme given by Algorithm 8, multiple occurrences of a word over different paragraphs can be caused not only by the node duplication, but by inheriting the word from parent to children.

In order to investigate how these two different schemes affect the dynamic correlations of Type-I words in a different manner, we adopted both of the two schemes to one tree structure simultaneously. That is, we always generated two pseudo-texts from one node sequence; one is the pseudo-text using the non-inheriting scheme (Algorithm 7) and the other is that using the inheriting scheme (Algorithm 8).

\section{Results of simulations}

\subsection{Number of frequent words with $\beta \leq \mathbf{0 . 8}$ and $\beta>0.8$}

We performed the simulations of generating pseudo-text independently 100 times. Thus, our whole simulations contained 100 times DAG generations each of which had 400 nodes, followed by converting the 100 DAGs to 100 tree structures, deriving 100 node sequences from the 100 tree structures and finally synthesizing 200 pseudo-documents from the 100 node sequences by the use of the inheriting and non-inheriting schemes. Results of the 100 times simulations are depicted in Fig. 12 in which the horizontal axes of the four plots represent the number of nodes in the converted tree structures. In plots (b), (c) and (d), circles $(O)$ represent the results of the inheriting scheme and squares $(\square)$ are those by the non-inheriting scheme.

As shown in Fig. 12a, the total number of pseudosentences in a pseudo-text is approximately proportional to the total number of nodes in the corresponding tree structure. This result is reasonable because we determined the number of sentences for each node by drawing from a Poisson distribution with a fixed mean 5 that is common to all pseudo-texts. Therefore, the proportional relationship between the number of pseudo-sentences and the number of nodes indicated by Fig. 12a convinces us that the drawings from a Poisson distribution worked well in our simulations.

The plot of Fig. $12 \mathrm{~b}$ shows the existence of a strong positive correlation between the number of nodes and the number of frequent words in a pseudo-text where we have used the same definition of the frequent word as our previous study [15], i.e., it is a word that occurs in more than or equal to 50 pseudo-sentences in a pseudotext. A close look of the distributions of the circles and the squares in Fig. 12b further indicates that there are more frequent words at the inheriting scheme than those at the non-inheriting one. As described before, some amount of words in the vocabulary are inherited from parents to children and children to grandchildren at the inheriting scheme. These inherited words are tended to occur more frequently and they are tended to become the frequent words. Therefore, the difference of the frequent word distribution between the two schemes shown in Fig. 12b can be interpreted as a verification for our algorithms of the inheriting and the non-inheriting schemes.

To investigate dynamic correlations of the frequent words in the 200 pseudo-texts, we calculated ACFs for all the frequent words in each of the 200 pseudo-texts and then performed curve fittings by the use of Eq. (6) for each calculated ACF. Through the fittings, we found that all of the ACFs can be reasonably fitted by Eq. (6); that is, all of the frequent words are Type-I words regardless whether they were generated by the non-inheriting or by the inheriting schemes. However, another important finding is that characteristic behaviors of ACFs at the non-inheriting and at the inheriting schemes are fundamentally different in the following manner. A plot of Fig. 12c demonstrates a distinctive difference between the two schemes in the number of frequent words having value of $\beta$ smaller than or equal to 0.8 ; at the inheriting scheme, there are $50-250$ frequent words with $\beta \leq 0.8$ in one pseudo-document, but at the non-inheriting scheme, the number of words with $\beta \leq 0.8$ is almost zero. In contrast, Fig. $12 \mathrm{~d}$ indicates that 
Fig. 12 Results of the simulations in which pseudo-texts were obtained from randomly generated original DAGs with 400 nodes. a Scatter plot of the number of nodes in tree structure versus the number of pseudo-sentences, b scatter plot of the number of nodes versus the number of frequent words in a pseudo-text, c scatter plot of the number of nodes versus the number of frequent words with $\beta \leq 0.8$ in a pseudo-text and $\mathbf{d}$ scatter plot of the number of nodes versus the number of frequent words with $\beta>0.8$ in a pseudo-text. In plots $\mathbf{b}$, c and $\mathbf{d}$, the each circle (O) represents the result of a pseudo-text derived from the inheriting scheme and the each square ( $\square$ ) is that from the non-inheriting scheme
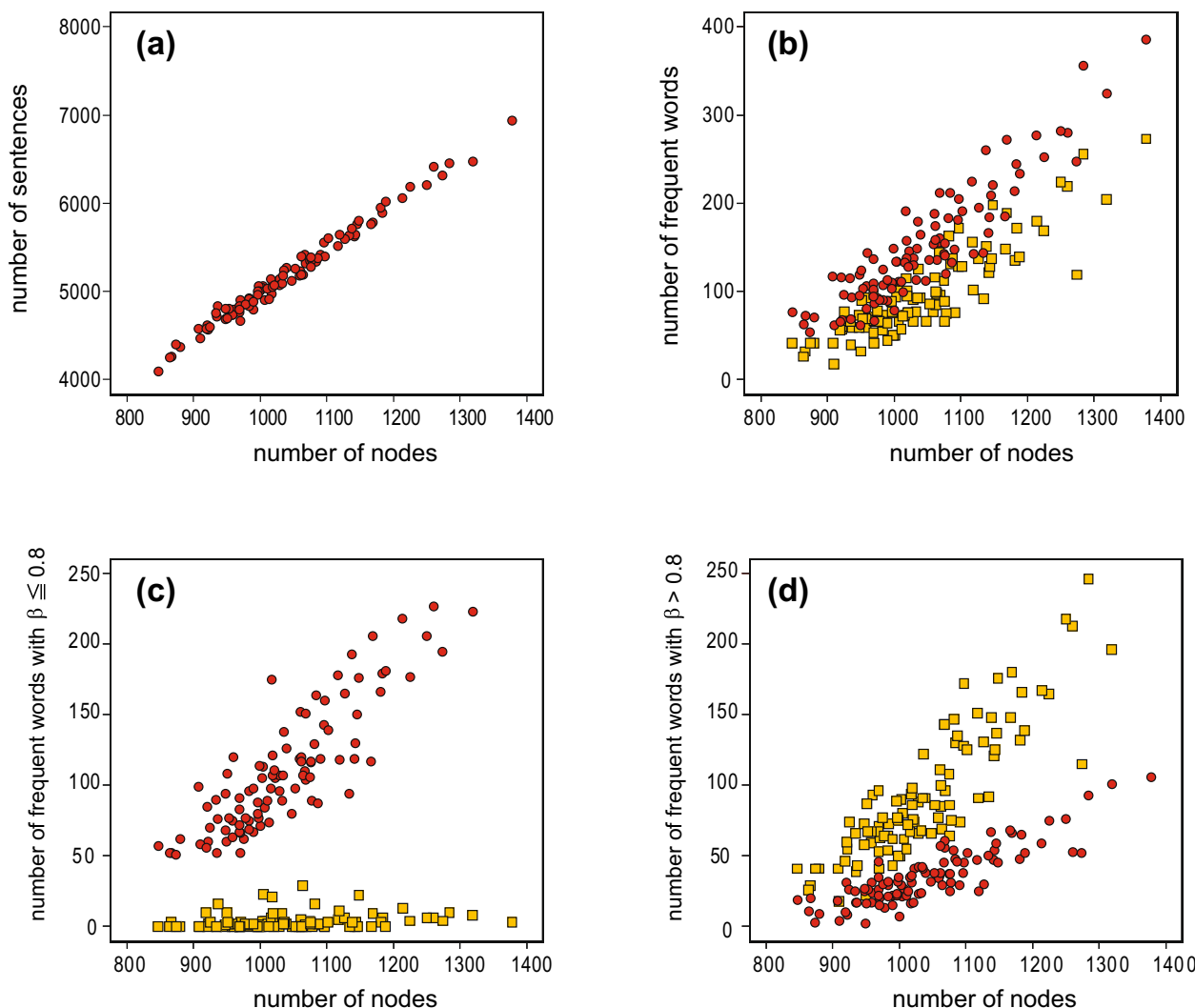

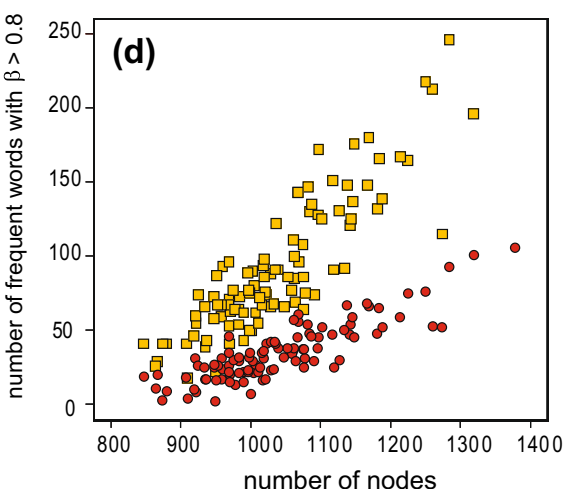

there are more frequent words with $\beta$ value larger than 0.8 at non-inheriting scheme than those at inheriting scheme. What is the reason that the frequent words with $\beta \leq 0.8$ are generated almost only in the inheriting scheme?

\section{2 $\beta$ and word index distributions}

To answer the question mentioned above, we closely examined how the frequent words are distributed in terms of word index and $\beta$ value. The results are shown in Fig. $13 \mathrm{a}$ and $\mathrm{b}$ for the non-inheriting and the inheriting schemes, respectively. Note that Fig. 13a and b are typical examples of plots for frequent words in two pseudodocuments that are generated from a same tree structure, but using different schemes. Figure 13a reveals that almost all of the frequent words are restricted in the region of $\beta>0.8$ and word index larger than 3500 for the noninheriting scheme. The reason that the word indices are restricted in the range above 3500 can be considered as follows. A requirement for a considered word to be a frequent word is that a node which the word belongs to is duplicated, because the word must occur among different paragraphs to be a frequent word and the multiple occurrences of a word over different paragraphs can take place only through the node duplication in the non-inheriting scheme, as described before. As intuitively indicated in Figs. 7 and 8, leaf nodes in an original DAG structure have the highest probability to be duplicated; that is, the probability of a considered node to be duplicated increases as the distance from the root to the considered node increases. Since the nodes that are distant from the root have characteristic words with larger word indices than the nodes near the root as a result of Algorithm 3, the indication of Fig. 13a is that the node duplications mainly occurred near or at the leaf nodes which have characteristic words with indices larger than 3500. Another point concerning about Fig. $13 \mathrm{a}$ is that about $70 \%$ of the frequent words are very densely plotted at $\beta=1$, i.e., 144 of the 204 frequent words have the ACFs with $\beta=1$. We will discuss the problem of this limited $\beta$ value at the non-inheriting scheme soon below, and the origin of the limited $\beta$ value will be attributed to the character of dynamic correlation at the non-inheriting scheme in which the duration time of dynamic correlation is limited within one paragraph.

In contrast to Fig. 13a, data points in Fig. 13b are widely scattered; $\beta$ is distributed from about 0.2 to 1.0 and word indices are distributed over its entire range. The most important result shown in Fig. 13b is that there is a clear tendency as indicated by dashed lines in which $\beta$ increases as increasing word index. This tendency can be interpreted in the following manner. Consider a word with a small word index. Since we used Algorithm 3 to assign words to nodes, the word with the small index should be originally assigned to a node near the root of the DAG structure. 
Fig. 13 Scatter plots of word index versus $\beta$ for a a sample pseudo-document generated with the non-inheriting scheme and $\mathbf{b}$ that generated with the inheriting scheme
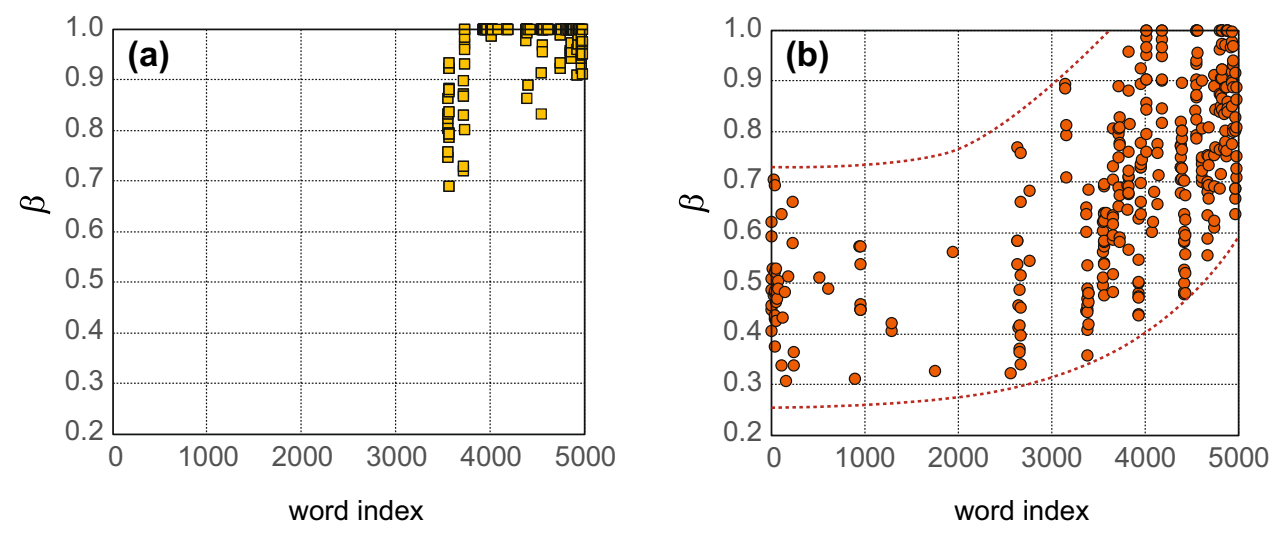

This word can be successively inherited from generation to generation multiple times, because Algorithm 8 assures the equal possibility of the word inheritance among all nodes in the node sequence and it also assures the inheritance can occur equally likely among all words in the vocabulary. If the word is inherited multiple times over several generations, then the word occurs many times in the pseudo-text and it becomes a frequent word. In this case, the duration time of the dynamic correlation of this word should extend to the entire range of the successive inheritance over several paragraphs and the complex correlation of the word among these paragraphs should be observed. Such a long-time and complex correlation is considered to be one major reason of ACFs with low $\beta$ values at the inheriting scheme because the correlation of this type brings in slower decay in ACFs than the decay of ACFs with $\beta=1$.

In short, a frequent word can be generated by two different mechanisms at the inheriting scheme: One is the inheritance over generations and the other is the duplication of nodes. Furthermore, we must consider the possibility of simultaneous occurrences of these two mechanisms. This can be occurred when a node which a considered word belongs to is duplicated and then the word is inherited from the original and duplicated nodes to their children. We can express this situation as several intervals in the node sequence in which the considered word is continuously used by successive inheritances. The first node of the first interval in the node sequence is the original node and the first nodes of other intervals are the duplicated nodes. Each interval has a length which is equal to the times of successive inheritances of the considered word. Since the lengths of the intervals are different with each other, this situation suggests that the characteristics of dynamic correlation of the considered word in each interval is different in the duration time of dynamic correlation and the ACF of the word becomes the average of these different correlations with the different duration times. This can be another reason of ACFs with low $\beta$ values because the superposition of ACFs with different duration times makes the resultant $\beta$ value lower [29].

We have shown in Fig. 5 that there are two components of $\beta$ distributions of Type-I words in real written texts. One is the skewed unimodal distribution peaked around $\beta=0.2$, and the other is a certain amount of population at $\beta=1.0$. From the consideration on the two different mechanisms of frequent word generation described above, we can attribute the two components in distribution as follows. The population at $\beta=1.0$ can be attributed to Type-I words that have short-range duration times of correlation which typically persists for several sentences. The dynamical correlations of such words correspond to those within one pseudo-paragraph in our simulations. The skewed unimodal distribution peaked around $\beta=0.2$ can be attributed to Type-I words that have long-range duration times which persists for several tens of sentences. These dynamical correlations with low $\beta$ values correspond to those due to the word inheritance among nodes or those due to the mixed effect of the word inheritance and the node duplication in our simulations.

\subsection{Difference in functional form of ACF}

In the following, we examine characteristic behaviors of ACFs at the non-inheriting and inheriting schemes through their functional forms and discuss the nature of dynamic correlations at each scheme in relation to the findings obtained from Fig. 13. Figure 14a shows a typical example of ACF from the non-inheriting scheme; it has $\beta=1$ and is for the word with index larger than 3500 . The word of which ACF is displayed in Fig. 14a is chosen from the frequent words plotted in Fig. 13a.

An important observation in Fig. 14a is that the ACF rapidly decreases to zero within lag step of 10 , which strongly suggests that the main behavior of the ACF at the non-inheriting scheme is governed by the correlation within one paragraph/node, because the averaged number of pseudo-sentences per node was set to be 5 
and the maximum number of that to be 10 in our simulations using Algorithm 5. To confirm that the correlation is limited within one paragraph at the non-inheriting scheme, we performed a modified simulation a result of which is shown in Fig. 14b. In the modified simulation, the number of pseudo-sentences for each node was also drawn from a Poisson distribution with mean value of 5 , but if the number drawn was larger than 5 , then it was reset to be 5 . Thus, the number of pseudo-sentences for each node was at most 5 , and the influence of this setting appears obviously at the dashed circle in Fig. 14b which indicates the abrupt decrease in the ACF to zero at lag step of 5 . We can therefore conclude that the dynamic correlation observed in the ACFs with $\beta=1$ and word index larger than 3500 , which occupy about $70 \%$ of all ACFs at the non-inheriting scheme as mentioned before, mainly arises from correlation within one paragraph.

Another point in Fig. 14a is that there are two characteristic humps as indicated by dashed circles. Such humps are commonly observed in ACFs from the non-inheriting scheme and can be attributed to fluctuations of ACFs due to occurrences of the node duplications at corresponding lag steps. For example, from the two humps at the lag steps about 40 and 80 in Fig. 14a, we can infer occurrences of two node duplications in the node sequence that occur at about 8th and 16th nodes later from the first appearance of the node including the considered word. ${ }^{3}$ Combining with the discussion about Fig. 13a in Sect. 4.2, we can conclude that, at the non-inheriting scheme, the node duplication serves a word to be a frequent word, but its influence on ACFs is quite limited because the influence appears only in supplementary humps and the major behavior of ACFs is determined by the correlation within one paragraph.

Figure $14 \mathrm{c}$ shows a sample ACF of a word chosen from the frequent words plotted in Fig. 13b which has low word index of 241 and hence inherited multiple times at the inheriting scheme. Since there is no abrupt decrease within lag step of 10 , we can infer that the duration of the dynamic correlation is not restricted within one paragraph and this is considered to be a consequence of the word inheritance over several generations giving longer duration than that restricted within one paragraph. Thus, the implication of the figure is that the dynamic correlation of the word is dominated not only by the correlation within paragraph, but by the correlations over paragraphs due to the word inheritance. As in Fig. 14a, the effect of node duplication is still supplementary, but appears as slight and random oscillation of the ACF in the case of Fig. 14c.
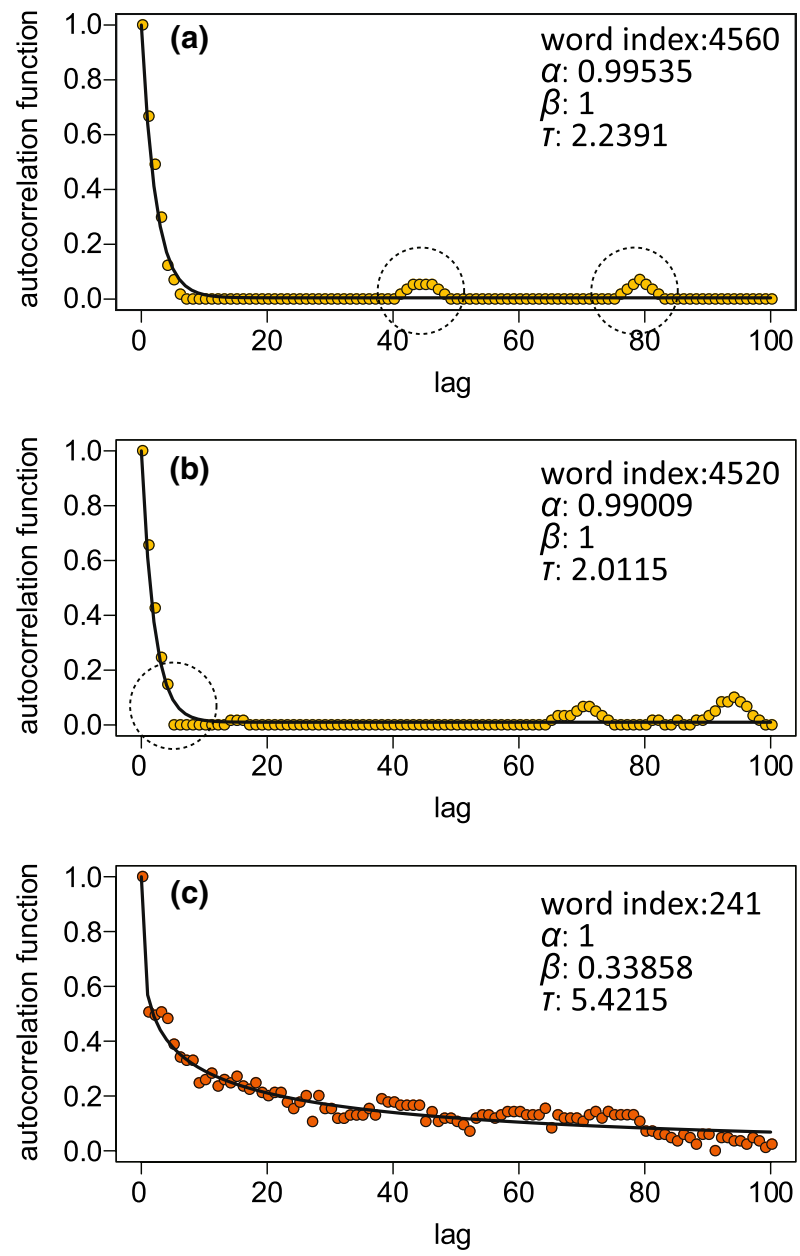

Fig. $14 \mathbf{a}$ and $\mathbf{b}$ : Calculated ACFs for words in pseudo-documents generated with non-inheriting scheme. The number of maximum pseudo-sentences per node is limited to be 10 in plot (a) and that is limited to be 5 in plot (b). c ACF for a word in a pseudo-document generated with inheriting scheme

\subsection{Summary of the simulation results}

The results of our simulation can be summarized as follows. Several tens to several hundreds of words out of 5000 words becomes Type-I words regardless of used schemes. The number of Type-I words at the inheriting scheme is, however, larger than that at the non-inheriting scheme because of the effect of the word inheritance. Therefore, as described in Sect. 4.2, a frequent word at the inheriting

\footnotetext{
3 Here, to calculate the positions of duplicated nodes, we have assumed that each node has 5 sentences which is actually the averaged number of sentences for each node. Of course, since the ACF expresses averaged behavior of dynamic correlations as seen in Eq. (5), the occurrences of node duplication at 8th and 16th node in the node sequence mentioned here should be also interpreted as a "averaged" behavior.
} 
scheme can be generated by two different mechanisms, i.e., the node duplication and the word inheritance. The role of the word inheritance to generate frequent words is clearly shown in Figs. $12 \mathrm{~b}$ and $13 \mathrm{~b}$ because Fig. $12 \mathrm{~b}$ indicates that the number of frequent words at the inheriting scheme is larger than that at the non-inheriting scheme and Fig. 13b indicates words with indices less than 3500 can be frequent words at the inheriting scheme, while frequent words with indices less than 3500 cannot be generated at the non-inheriting scheme.

At the non-inheriting scheme, almost all of the ACFs of the Type-I words have $\beta$ larger than 0.8 and about $70 \%$ of the ACFs have $\beta=1$. It is turned out that the dynamic correlation of such ACFs as in Fig. 14a arises mainly from the correlation within one paragraph.

At the inheriting scheme, the word inheritance plays a central role in generating characteristic ACFs. The inheritance extends the duration time of the dynamic correlation to successive paragraphs wherein the inheritance of a considered word continuously occurs. It also makes the correlation of the considered word more complex because the correlation within one paragraph and that between paragraphs are superposed to form ACFs as in Fig. 14c. The effects of the duration time extension and the complex correlation among successive paragraphs on $\beta$ can be recognized from the comparison between Figs. 13a and b. For example, we can compare the distribution of $\beta$ values in Fig. 13a with those in Fig. 13b around a specified word index. If we restrict word indices near 5000 , then $\beta$ takes values from about 0.9 to 1 in Fig. 13a, while it takes values from about 0.6 to 1.0 in Fig. 13b, which clearly suggests that the occurrences of the word inheritance make $\beta$ lower.

\section{Conclusion}

To gather information on the origin of Type-I words, we conducted simulations of generating a pseudo-text. In the simulation procedures, we tried to trace the conversion process from our thought/idea into a written text in the following manner. We first assumed that our thought/ idea to be expressed in a written text can be described by a directed acyclic graph (DAG). Each topic to be involved in the written text corresponded to the each node in the DAG, and the each node was assumed to have characteristic unique words to describe the topic. We further assumed that the conversion process can be traced by the conversion of the DAG to tree structure followed by a derivation of the node sequence from the tree structure. The order of topics to be described in pseudo-text was determined by node sequence which was derived using the depth-first search algorithm. Then the pseudo-paragraph was generated from each node in the node sequence using two different schemes. One was that called the non-inheriting scheme where the pseudo-paragraph was synthesized only from the originally assigned characteristic words to the corresponding node and the other was that called the inheriting scheme where the pseudo-paragraph was synthesized from both the originally assigned characteristic words and inherited words from a parent node in the tree structure. The simulation results showed that the both schemes can generate Type-I words, but the inheriting scheme only gives the ACFs with strong dynamical correlation which are seen typically for the important words in real written texts. This result indicates that the long-range word correlation embedded in the inheriting scheme due to the word inheritance plays the central role in reproducing the long-range correlation among several to several tens of sentences in real written texts.

Acknowledgements We thank Dr. Yusuke Higuchi for useful discussion and illuminating suggestions. This work was supported in part by JSPS Grant-in-Aid (Grant Nos. 25589003 and 16K00160).

\section{Compliance with ethical standards}

Conflict of interest The authors declare that there is no conflict of interest of any sort for this research.

\section{Appendix: Summary of English texts employed}

In this study, we selected the English edition of 12 famous books as samples of written texts and analyzed all Type-I words appearing therein to clarify the features of Type-I words. They are listed in Table 1 with their short names and some information. Five of them are popular novels (Carroll, Twain, Austen, Tolstoy and Melville) and the rest are chosen from the categories of natural science (Darwin, Einstein and Lavoisier), psychology (Freud), political economy (Smith) and philosophy (Kant and Plato), so as to represent a wide range of written texts. The books were downloaded as text files from Project Gutenberg (https ://www.gutenberg.org). The preface, contents and index pages were deleted before starting text preprocessing because they may act as noise and may affect the final results [15].

Some basic statistics of the used texts, evaluated after the preprocessing procedures, are listed in Table 2 . The heading "frequent word" at the last column of the table indicates that words listed in the column appeared in at least 50 sentences in the relevant text. 
Table 1 Summary of English texts employed

\begin{tabular}{llll}
\hline Short name & Title & Author & Download URL \\
\hline Carroll & Alice's Adventures in Wonderland & Lewis Carroll & https://www.gutenberg.org/ebooks/11 \\
Twain & The Adventures of Tom Sawyer & Mark Twain & https://www.gutenberg.org/ebooks/74 \\
Austen & Pride and Prejudice & Jane Austen & https://www.gutenberg.org/ebooks/1342 \\
Tolstoy & War and Peace & Leo Tolstoy & https://www.gutenberg.org/ebooks/2600 \\
Melville & Moby Dick; Or, The Whale & Herman Melville & https://www.gutenberg.org/ebooks/2701 \\
Darwin & On the Origin of Species & Charles Darwin & https://www.gutenberg.org/ebooks/1228 \\
Einstein & Relativity: The Special and General Theory & Albert Einstein & https://www.gutenberg.org/ebooks/5001 \\
Lavoisier & Elements of Chemistry & Antoine Lavoisier & https://www.gutenberg.org/ebooks/30775 \\
Freud & Dream Psychology & Sigmund Freud & https://www.gutenberg.org/ebooks/15489 \\
Smith & An Inquiry into the Nature and Causes of the & Adam Smith & https://www.gutenberg.org/ebooks/3300 \\
& Wealth of Nations & & \\
Kant & The Critique of Pure Reason & Immanuel Kant & https://www.gutenberg.org/ebooks/4280 \\
Plato & The Republic & Plato & https://www.gutenberg.org/ebooks/1497 \\
\hline
\end{tabular}

Table 2 Basic statistics of the 12 texts, evaluated after preprocessing procedures

\begin{tabular}{lcrrc}
\hline Text & $\begin{array}{l}\text { Vocabulary } \\
\text { size }\end{array}$ & $\begin{array}{l}\text { Length in } \\
\text { words }\end{array}$ & $\begin{array}{l}\text { Length in } \\
\text { sentences }\end{array}$ & $\begin{array}{l}\text { Number of } \\
\text { frequent } \\
\text { words }\end{array}$ \\
\hline Carroll & 1848 & 8191 & 1098 & 10 \\
Twain & 5981 & 25,682 & 4288 & 46 \\
Austen & 4643 & 39,590 & 5523 & 136 \\
Tolstoy & 14,555 & 212,483 & 28,432 & 797 \\
Melville & 14,413 & 85,557 & 8556 & 237 \\
Darwin & 5316 & 58,611 & 3991 & 212 \\
Einstein & 1893 & 11,642 & 963 & 24 \\
Lavoisier & 3841 & 42,417 & 3029 & 155 \\
Freud & 4006 & 19,533 & 1828 & 30 \\
Smith & 6817 & 140,905 & 11,318 & 537 \\
Kant & 5792 & 75,285 & 5715 & 289 \\
Plato & 5400 & 35,491 & 4468 & 103 \\
\hline
\end{tabular}

\section{References}

1. Alvarez-Lacalle E, Dorow B, Eckmann JP, Moses E (2006) Hierarchical structures induce long-range dynamic correlations in written texts. Proc Natl Acad Sci USA 103(21):7956-7961

2. Altmann EG, Cristadoro G, Esposti MD (2012) On the origin of long-range correlations in texts. Proc Natl Acad Sci USA 109(29):11582-11587

3. Chatzigeorgiou M, Constantoudis V, Diakonos F, Karamanos K, Papadimitriou C, Kalimeri M, Papageorgiou H (2017) Multifractal correlations in natural language written texts: effects of language family and long word statistics. Physica A 469:173-182

4. Schenkel A, Zhang J, Zhang Y (1993) Long range correlation in human writings. Fractals 01(01):47-57

5. Ebeling W, Pöschel T (1994) Entropy and long-range correlations in literary english. Europhys Lett 26(4):241

6. Montemurro MA, Pury PA (2002) Long-range fractal correlations in literary corpora. Fractals 10(04):451-461
7. Sarkar A, Garthwaite P, De Roeck A (2005) A bayesian mixture model for term re-occurrence and burstiness. In: Ninth conference on computational language learning (CoNLL), pp 29-30

8. Altmann EG, Pierrehumbert JB, Motter AE (2009) Beyond word frequency: bursts, lulls, and scaling in the temporal distributions of words. PLoS ONE 4(11):e7678. https://doi.org/10.1371/journ al.pone. 0007678

9. Perakh M (2012) Serial correlation statistics of written texts. Int J Comput Linguist Appl 3:11-43

10. Tanaka-Ishii K, Bunde A (2016) Long-range memory in literary texts: on the universal clustering of the rare words. PLoS ONE 11(11):e0164658. https://doi.org/10.1371/journal.pone.01646 58

11. National Institute of Standards and Technology (2013) e-Handbook of Statistical Methods. http://www.itl.nist.gov/div898/ handbook/

12. Frenkel I, Gertsbakh IB, Khvatskin LV (2003) Parameter estimation and hypotheses testing for nonhomogeneous poisson process. Transp Telecommun 4(2):9-17

13. Tijms HC (2003) A first course in stochastic models. Wiley, Hoboken

14. Ross SM (1996) Stochastic processes. In: Wiley series in probability and statistics. Wiley

15. Ogura $\mathrm{H}$, Amano $\mathrm{H}$, Kondo M (2019) Measuring dynamic correlations of words in written texts with an autocorrelation function. J Data Anal Inf Process 7(2):46-73

16. Ogura $H$, Amano $H$, Kondo $M$ (2019) Origin of dynamic correlations of words in written texts. J Data Anal Inf Process 7(4):228-249

17. Palmer RG, Stein DL, Abrahams E, Anderson PW (1984) Models of hierarchically constrained dynamics for glassy relaxation. Phys Rev Lett 53(10):958-961

18. Kakalios J, Street RA, Jackson WB (1987) Stretched-exponential relaxation arising from dispersive diffusion of hydrogen in amorphous silicon. Phys Rev Lett 59(9):1037-1040

19. Milovanov AV, Rasmussen JJ, Rypdal K (2008) Stretched-exponential decay functions from a self-consistent model of dielectric relaxation. Phys Lett A 372(13):2148-2154

20. Papoulis A, Pillai SU (2002) Probability, random variables and stochastic processes. McGraw-Hill, New York

21. Hsu HP (1997) Probability, random variables, and random processes. McGraw-Hill, New York 
22. Elton DC (2018) Stretched Exponential Relaxation. ArXiv e-prints, August 2018

23. Markelov OA, Nguyen VD, Mikhail B (2017) Statistical modeling of the internet traffic dynamics: to which extent do we need long-term correlations? Physica A 485:48-60

24. Bogachev MI, Markelov OA, Kayumov AR, Bunde A (2017) Superstatistical model of bacterial DNA architecture. Sci Rep 7:43034. https://doi.org/10.1038/srep43034

25. Nguyen VD, Bogachev MI, Markelov OA, Vasenev AN (2017) Superstatistical description of aggregated network traffic with two-compound access rate distribution. In: 2017 XX IEEE international conference on soft computing and measurements (SCM). St. Petersburg, pp 57-60. https://doi.org/10.1109/ SCM.2017.7970494

26. Symeonidis A, Tollis IG, Reczko M (2006) Visualization of functional aspects of microrna regulatory networks using the gene ontology. In: 7th international symposium biological and medical data analysis, ISBMDA, pp 13-24

27. Levitin A (2011) Introduction to the design and analysis of algorithms, 3rd edn. Pearson, London

28. Bishop CM (2006) Pattern recognition and machine learning, 1st edn. Springer, Berlin

29. Berberan-Santos MN, Bodunov EN, Valeur B (2005) Mathematical functions for the analysis of luminescence decays with underlying distributions 1 kohlrausch decay function (stretched exponential). Chem Phys 315(1-2):171-182

Publisher's Note Springer Nature remains neutral with regard to jurisdictional claims in published maps and institutional affiliations. 